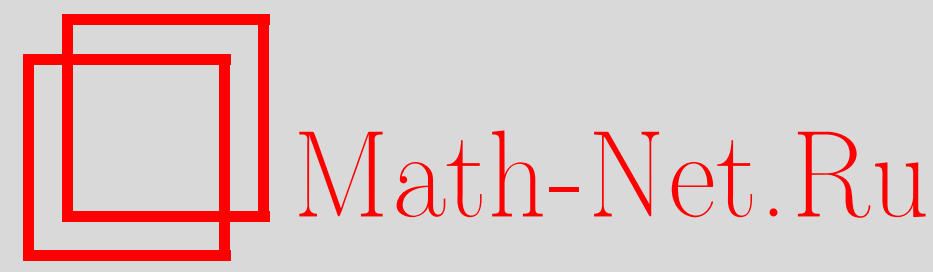

В. С. Матвеев, Интегрируемые гамильтоновы системы с двумя степенями свободы. Топологическое строение насыщенных окрестностей точек типа фокус-фокус и седло-седло, Матем. сб., 1996, том 187, номер 4, 29-58

DOI: https://doi.org/10.4213/sm122

Использование Общероссийского математического портала Math-Net.Ru подразумевает, что вы прочитали и согласны с пользовательским соглашением

http://www . mathnet.ru/rus/agreement

Параметры загрузки:

IP: 54.84 .234 .179

26 апреля 2023 г., 12:13:47 
УДК 514.7

\author{
В.С. Матвеев
}

\title{
Интегрируемые гамильтоновы
} системы с двумя степенями свободы.

\section{Топологическое строение насыщенных окрестностей точек типа фокус-фокус и седло-седло}

\begin{abstract}
Одной из ключевых задач гамильтоновой механики является описание поведения интегрируемых гамильтоновых систем с двумя степенями свободы в окрестностях особых слоев слоения Лиувилля. В 1988 году Л. М. Лерман и Я. Л. Уманский анонсировали теорему классификации таких систем для случая, когда особый слой содержит одну точку ранга 0. В 1992 и 1993 годах доказательство было опубликовано. При решении классических задач физики и механики возникают интегрируемые гамильтоновы системы с несколькими точками ранга 0 на особом слое. Настоящая работа посвящена топологической классификации интегрируемых гамильтоновых систем в окрестности особых слоев, содержащих произвольное число точек ранга 0 .

Библиографоия: 12 названий.
\end{abstract}

\section{§1. Введение}

Под интегрируемой гамильтоновой системой мы будем понимать четверку $\left(M^{4}, \omega, h, f\right)$, где $\left(M^{4}, \omega\right)$ - симплектическое многообразие, $h$ и $f$-гладкие, находяшиеся в инволюции функции на $M^{4}$. Предполагаем, что $f$ и $h$ функционально независимы, т.е. дифференциалы функций $f$ и $h$ независимы почти всюду, и что совместные поверхности уровня функций $f^{-1}(a) \cap h^{-1}(b)$ компактны для любых чисел $a$ и $b$.

Такая ситуация естественно возникает, например, в классической механике. Симплектическим многообразием $\left(M^{4}, \omega\right)$ в этом случае может являться кокасательное расслоение к конфигурационному пространству с естественной симплектической структурой, функцией $h$ - гамильтониан, а функцией $f$ - первый интеграл системы. В некоторых реальных интегрируемых гамильтоновых системах симплектическим многообразием является орбита коприсоединенного представления со скобкой Пуассона-Ли на ней.

Определим отображение момента $\varkappa: M^{4} \rightarrow \mathbb{R}^{2}$ по правилу $\varkappa(x)=(h(x), f(x))$. Это отображение задает так называемое слоение Лиувилля многообразия $M^{4}$. Слоями являются связные компоненты поверхности уровня отображения $\varkappa$. Напомним, что по теореме Лиувилля любой неособый слой гомеоморфен двумерному тору.

Будем называть замкнутую простую кривую критической окружностью, если векторные поля $\operatorname{sgrad}_{f}$ и $\operatorname{sgrad}_{h}$ касаются ее в каждой точке. Если векторное поле $\operatorname{sgrad}_{h}$ не обрашается в нуль на критической окружности, то ориентируем 
критическую окружность векторньм полем $\operatorname{sgrad}_{h}$, в противном случае оставим критическую окружность неориентированной.

Интегрируемые гамильтоновые системы $\left(M^{4}, \omega, h, f\right)$ и $\left(M_{1}^{4}, \omega_{1}, h_{1}, f_{1}\right)$ будем называть топологически әквивалентными, если между многообразиями $M^{4}$ и $M_{1}^{4}$ существует гомеоморфизм, сохраняющий слоение Лиувилля и переводящий критические окружности в критические окружности с сохранением ориентации.

В работах [1], [2], [9] фактически дана полная топологическая классификация в достаточно тонкой регулярной окрестности устойчивой невырожденной изоэнергетической поверхности $Q_{3}$. Легко видеть, что топологическое строение такой системы получается из топологического строения $Q_{3}$ прямым умножением на отрезок (здесь и далее слоями на прямом произведении множсеств считаем всевозможные прямые произведения слоев на компонентах, слоями на отрезке в данном случае являются точки). В работе [1] по изоэнергетической поверхности $Q_{3}$ строится ориентированный граф $W\left(Q_{3}\right)$, вершинам которого сопоставлены так называемые атомы. Молекула $W\left(Q_{3}\right)$ классифицирует изоэнергетические поверхности с точностью до так называемой “грубой топологической” эквивалентности. В работе [2] ребра и вершины этого графа оснащаются некоторыми рациональными метками так, что полученная в результате меченая молекула $W^{*}\left(Q_{3}\right)$ классифицирует устойчивые изоэнергетические поверхности с точностью до топологической эквивалентности. В работе [9] доказана теорема реализации.

Комментарий. А.Н. Ошемков, А.В. Болсинов, П.И. Топалов и другие построили меченые молекулы для отдельных изоэнергетических поверхностей интегрируемых гамильтоновых систем, встречающихся в задачах описания движения твердого тела. Оказалось, что, по крайней мере для таких интегрируемых гамильтоновых систем, меченые молекулы достаточно емко описьвают топологическое строение всей системы.

Бифуркационной диаграммой будем называть множество $\Sigma=\varkappa\left\{x \in M^{4}\right.$ : $\left.\left.d \varkappa\right|_{x}<2\right\}$ критических значений отображения момента. Как правило, бифуркационная диаграмма состоит из конечного набора гладких кривых и изолированных точек, причем число точек пересечения и касания кривых конечно.

Заметим, что неустойчивость изоэнергетической поверхности тесно связана с невозможностью представить бифуркационную диаграмму в окрестности соответствующей изоэнергетической поверхности вертикальной прямой в виде прямого произведения дискретного набора точек на отрезок.

Пусть бифуркационная диаграмма в окрестности точки $A$ имеет вид, изображенный на рис. 1 а)-г). Предполагаем, что прообраз точки $A$ связен. Такая ситуация обычно возникает, если в прообразе точки $A$ есть невырожденная особая точка ранга нуль.

Для этой работы особый интерес представляют исследования Л.М. Лермана и Я. Л. Уманского. В своих работах [3], [4], см. также [11], они ввели понятие невырожденности для точек ранга нуль и разбили невырожденные точки ранга нуль на 4 типа: центр-центр, центр-седло, седло-седло и фокус-фокус. Бифуркационная диаграмма 1 а) отвечает точке типа центр-центр, 1 б) - центр-седло, 1 в) - седло-седло и 1 г) - фокус-фокус.

Определения невырожденности точек и типов точек смотри в $\S 2$. 

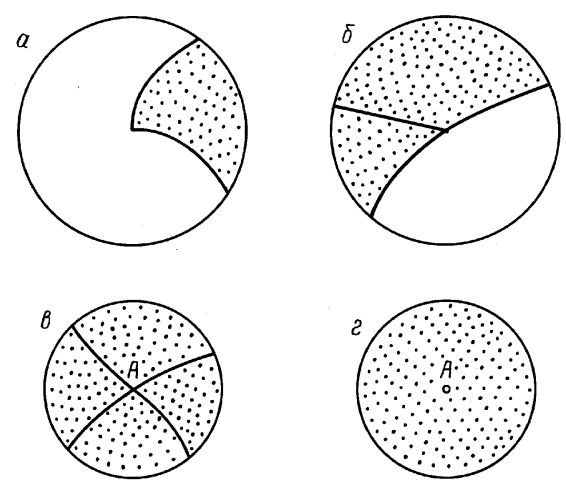

Рис. 1

Насыщенной окрестностью мы будем называть связную окрестность, целиком состоящую из слоев расслоения Лиувилля.

Случаи а) и б) достаточно хорошо изучены. Известно, см., например, [6], [10], что малая насышенная окрестность точки типа центр-центр топологически эквивалентна прямому произведению $\left\{D_{2}, f\right\} \times\left\{D_{2}, f\right\}$, где $D_{2}=\left\{x^{2}+y^{2}<1\right\}$ - двумерный диск, слоение на котором задается функцией $f=x^{2}+y^{2}$. А.В. Болсинов в работе [5] показал, что множество малых насыщенных окрестностей точек типа центр-седло с точностью до топологической эквивалентности параметризуется множеством ориентируемых букв-атомов. Причем буква-атом $\left\{P_{2}, h\right\}$ соответствует малой насыщенной окрестности точки типа центр-седло $\left\{P_{2}, h\right\} \times\left\{D_{2}, f\right\}$.

Данная работа посвящена топологическому описанию насыщенных окрестностей точек типа седло-седло и фокус-фокус.

Пусть $\mathfrak{U}_{S}$ - насыщенная окрестность точки типа седло-седло, причем:

1) бифуркационная диаграмма ограничения отображения момента на $\mathfrak{U}_{S}$ состоит из пары пересекающихся (по точке $A$ ) интервалов, каждый из интервалов есть график гладкой функции от $h$ (рис. 1 в));

2) $\mathfrak{U}_{S}$ не содержит вырожденных особых точек;

3) прообраз точки $A$ связен, $\mathfrak{U}_{S}$ является регулярной окрестностью прообраза $\varkappa^{-1}(A)$.

Такие окрестности будем называть седловыми. Из $§ 3$ будет следовать, что если все точки ранга нуль некоторого слоя невырожденны, то некоторая насышенная окрестность этого слоя не содержит вырожденных особых точек. Можно показать, что любые две седловые окрестности одной и той же точки типа седло-седло топологически эквивалентны.

Пусть $\mathfrak{U}_{F}$ - насышенная окрестность точки типа фокус-фокус, причем бифуркационная диаграмма ограничения отображения момента $\varkappa$ на $\mathfrak{U}_{F}$ состоит из одной точки $A$ (рис. 1 г)), и прообраз $\varkappa^{-1}(A)$ связен и не содержит вырожденных точек. Такие окрестности будем называть фокусными. Все фокусные окрестности одной и той же точки типа фокус-фокус топологически эквивалентны. 
В дальнейшем мы будем предполагать, что седловые и фокусные окрестности заданы в финитных терминах, позволяюших

а) эффективно строить множества, определяемые уравнениями $\operatorname{rank}(d \varkappa)<2$,

б) эффективно вычислять образ и прообраз любого финитно заданного множества,

в) эффективно строить пересечение любых множеств.

В частности, седловые и фокусные окрестности, встречаюшиеся при решении классических задач механики и физики, удовлетворяют этим условиям.

\section{Постановка проблем.}

1) Проблема распознавания. Существует ли алгоритм, который по двум седловьг или фокусным окрестностям определяет, эквивалентны ли они.

2) Проблема перечисления. Существует ли алгоритм, перечисляющий все седловые и фокусные окрестности с точностью до топологической эквивалентности.

3) Проблема описания топологического строения гранишы. Существует ли алгоритм, который по седловой или фокусной окрестности описывает топологическое строение границы этой окрестности.

ЗАмЕчАНИЕ. Проблема описания топологического строения границы интересна в связи с гипотезой Фоменко о круговых меченых молекулах. Пусть $B$ - точка на бифуркационной диаграмме, прообраз $\varkappa^{-1}(B)$ связен и содержит точки ранга нуль. Рассмотрим маленькую окружность $\gamma$ на бифуркационной диаграмме с центром в точке $B$. Прообраз $\varkappa^{-1}(\gamma)$ - трехмерное многообразие. Топологическое строение слоения Лиувилля этого многообразия может быть описано в терминах меченой молекулы.

Круговой меченой молекулой точки $B$ назовем меченую молекулу $W^{*}\left(\varkappa^{-1}(\gamma)\right)$. Заметим, что круговая меченая молекула точки $A$ есть в точности описание топологического строения гранищы в терминах меченых молекул.

ПроБлема (А.Т. Фоменко). Верно ли, что круговая меченая молекула точки В есть полный топологический инвариант достаточно тонкой насъценной регулярной окрестности слоя $\varkappa^{-1}(B)$.

КомментА Рй̆. Предполагается, что на интегрируемую гамильтонову систему наложены некоторые ограничения. Например, естественно предполагать, что топологическое строение прообраза окружности стабилизируется при уменьшении радиуса окружности. Более того, возникает проблема нахождения наиболее широкого в некотором смысле класса интегрируемых гамильтоновых систем, для которого проблема Фоменко верна. В него заведомо входят системы, содержащие особые точки только типа центр-центр и центр-седло, [3], [5], [6] .

\section{Формулировка результатов.}

ОСНОВНАЯ ТЕОРЕМА. Все три перечисленные проблемы имеют положительное конструктивное решение. А именно:

1) существует алгоритм, который по двум седловым или фокусным окрестностям определяет, әквивалентны ли они;

2) существует алгоритм, перечисляющий все седловые и фокуснье насыщенные окрестности с точностью до топологической эквивалентности; 
3) существует алгоритм, который по седловой или фокусной окрестности описывает топологическое строение границы этой окрестности.

Теоремы о проблеме Фоменко. Весом седловой или фокусной окрестности назовем число точек ранга 0 в ней.

Пусть $\mathfrak{U}_{S}$ - седловая окрестность, точка $A_{S}$ - точка пересечения линий бифуркационной диаграммы.

ТЕОРЕМА О КРУГОВЫХ МОЛЕКУЛАХ СЕДЛОВЫХ ОКРЕСТНОСТЕЙ. Круговая молекула точки $A_{S}$ есть полный топологический инвариант седловьг окрестностей веса 1,2 и 3.

Пусть $\mathfrak{U}_{F}-$ фокусная окрестность, точка $A_{F}$ - бифуркационная диаграмма $\mathfrak{U}_{F}$.

ТЕОРЕМА О КРУГОВЫХ МОЛЕКУЛАХ ФОКУСНЫХ ОКРЕСТНОСТЕЙ. Круговая молекула точки $A_{F}$ есть полный топологический инвариант фокусных окрестностей.

В $\S 2$ даны основные определения и доказаны технические леммы о невырожденных орбитах. В $\S 3$ строится новый топологический инвариант для седловых окрестностей - CL-тип - и, тем самым, решается проблема распознавания для них. В $\S 4$ доказывается теорема реализации, тем самым, решается проблема перечисления. $\S 5$ посвящен проблеме описания топологического строения границы седловой окрестности. В нем доказывается теорема о гипотезе Фоменко для седловых окрестностей. В $\S 6$ решаются все три проблемы для фокусных окрестностей. В $\S 7$ обобшается информация о гипотезе Фоменко, топологически описываются седловые и фокусные окрестности, встретившиеся в физических интегрируемых гамильтоновых системах и рассматриваются некоторые обобшения CL-типа.

В заключение автор пользуется случаем поблагодарить А. Т. Фоменко за постоянное внимание к работе и А.В. Болсинова за ценные обсуждения и замечания, позволившие существенно улучшить работу.

\section{§ 2. Особые невырожденные орбиты}

В этом параграфе будут даны определения особых невырожденных одномерных и нульмерных орбит и описаны их простейшие свойства.

Пусть $\left(M^{4}, \omega, h, f\right)$ - интегрируемая гамильтонова система. Введем на $M^{4}$ два гладких векторных поля $\operatorname{sgrad}_{h}$ и $\operatorname{sgrad}_{f}$. Они однозначно определяются соотношениями $\omega\left(\operatorname{sgrad}_{h}, v\right)=v(h), \omega\left(\operatorname{sgrad}_{f}, v\right)=v(f)$, где $v$ обозначает произвольное векторное поле на $M^{4}$, а $v(*)$ - производную функции вдоль него. Эти два векторных поля коммутируют, так как, по определению, $\omega\left(\operatorname{sgrad}_{h}, \operatorname{sgrad}_{f}\right)=\{h, f\}=0$. Поэтому можно определить действие Пуассона группы $\mathbb{R}^{2}$ на $M^{4}$, порожденное сдвигами вдоль интегральных траекторий полей $\operatorname{sgrad}_{h}$ и $\operatorname{sgrad}_{f}$. Легко показать, что сдвиги вдоль полей $\operatorname{sgrad}_{h}$ и $\operatorname{sgrad}_{f}$ сохраняют функции $h, f$ и форму $\omega$.

ЛЕмма 1 (о коэффициентах зависимости между $d h$ и $d f$ на орбите). Пусть $x \in$ $M^{4}, \theta(x)$ - орбита точки $x$. Пусть для некоторьх $\lambda$ и $\left.\mu(\lambda d h+\mu d f)\right|_{x}=0$. $\left.\operatorname{Toгдa~}(\lambda d h+\mu d f)\right|_{\theta(x)}=0$. 
Доказательство очевидно, так как сдвиги вдоль векторных полей $\operatorname{sgrad}_{h}$ и $\operatorname{sgrad}_{f}$ являются диффеоморфизмами, сохраняюшими функцию $\lambda h+\mu f$. Поэтому

$$
\left.d(\lambda h+\mu f)\right|_{x}=\left.d(\lambda h+\mu f)\right|_{\theta(x)}=0 .
$$

ОПРЕДЕЛЕНИЕ. Рангом точки $x \in M^{4}$ назовем ранг дифференциала отображения момента $\varkappa$ в этой точке: $\operatorname{rank}(x)=\operatorname{rank}(d \varkappa(x))$.

Приведем два полезных следствия леммы 1.

СлеДСтвиЕ 1. Пусть $\operatorname{rank}(x)=1$. Тогда орбита $\theta(x)$ одномерна.

СлЕДСТвИЕ 2. Пусть $\operatorname{rank}(x)=0$. Тогда орбита $\theta(x)$ состоит из одной точки $x$.

Невырожденные одномерные орбиты. Пусть $x$ - точка ранга 1 . Это означает, что существует такая ненулевая пара действительных чисел $(\lambda, \mu)$, что $\left.(\lambda d h+\mu d f)\right|_{x}=0$ и хотя бы один дифференциал нулю не равен.

Дадим два эквивалентных определения невырожденности одномерных орбит: геометрическое и аналитическое.

Пусть $x \in M^{4}$ и $\left.(\lambda d h+\mu d f)\right|_{x}=0$. Для определенности считаем, что $\operatorname{sgrad}_{h}$ в точке $x$ не равен нулю. Рассмотрим небольшую окрестность $\Upsilon_{x}$ точки $x$ и трехмерную поверхность $\Theta_{h}$ в ней, задаваемую уравнением $h=\mathrm{const}=h(x)$. На поверхности $\Theta_{h}$ векторное поле $\operatorname{sgrad}_{h}$ невырожденно. Рассмотрим двумерную трансверсаль к нему, проходяшую через точку $x$. Обозначим ее через $B_{x}$.

ГЕОМЕТРИЧЕСКОЕ ОПРЕДЕЛЕНИЕ. Назовем орбиту $\theta(x)$ невырожденной, если особенность ограничения функции $f$ на $B_{x}$ морсовская.

Легко видеть, что свойство функции $f$ быть морсовской на трансверсали к векторному полю $\operatorname{sgrad}_{h}$ не зависит от выбора трансверсали в точке $x$.

Сдвигом вдоль векторного поля $\operatorname{sgrad}_{h}$ можно перевести окрестность точки $x$ в окрестность любой точки орбиты. Такой сдвиг сохраняет функции $h, f$, форму $\omega$ и трансверсаль переводит в трансверсаль. Следовательно, определение невырожденности одномерной орбиты корректно.

Дадим аналитическое определение невырожденности.

По теореме Дарбу в окрестности $\Upsilon_{x}$ существуют симплектические координаты $p_{1}, q_{1}, p_{2}, q_{2}$ такие, что $p_{1}(y)=h(y)-h(x), y \in \Upsilon_{x}$. В них $\frac{\partial f}{\partial q_{1}}=0$.

АНАЛИТИЧЕСКОЕ ОПРЕДЕЛЕНИЕ. Назовем орбиту $\theta(x)$ невырожденной, если определитель

отличен от нуля.

$$
\Delta=\operatorname{det}\left|\begin{array}{cc}
\frac{\partial^{2} f}{\partial p_{2}^{2}} & \frac{\partial^{2} f}{\partial p_{2} \partial q_{2}} \\
\frac{\partial^{2} f}{\partial q_{2} \partial p_{2}} & \frac{\partial^{2} f}{\partial q_{2}^{2}}
\end{array}\right|
$$

Геометрическое и аналитическое определения невырожденности одномерных орбит эквивалентны, так как условия $p_{1}=0, q_{1}=0$ задают двумерную трансверсаль к потоку векторного поля $\operatorname{sgrad}_{h}$ на гиперповерхности $\{h=\mathrm{const}=h(x)\}$.

Если определитель $\Delta$ положительный, то орбиту будем называть әллиптической, иначе седловой. 
ЛЕмма 2 (о топологическом строении трубчатой окрестности седловой орбиты). Пусть $\theta(x)$ - отрезок одномерной седловой орбить. Тогда у него существует регулярная окрестность $R_{\theta(x)}$, топологически эквивалентная четырехмерному диску $\left\{\left|p_{1}\right|<1,\left|q_{1}\right|<1,\left|p_{2} q_{2}\right|<1,\left|p_{2}\right|<1,\left|q_{2}\right|<1\right\}$, расслоенному на связнье компоненты совместных поверхностей уровня функиий $f=p_{2} q_{2}$ и $h=p_{1}$. Более того, существует диффеоморфизм, сохраняющий слоение Лиувилля между $R_{\theta(x)}$ и этим диском.

Удобно представлять себе $R_{\theta(x)}$ как прямоепроизведение $\left\{S^{2}, f\right\} \times\left\{I_{1}, h\right\} \times\left\{I_{2}\right\}$, где $S^{2}=\left\{\left|p_{1} q_{1}\right|<1 / 2,\left|p_{1}\right|<1,\left|q_{1}\right|<1\right\}$ - двумерньй диск, расслоенный функцией $f=p_{1} q_{1}$ на связные компоненты ее поверхности уровня, $p_{1}$ и $q_{1}-$ декартовы координаты на плоскости (рис. 3). $I_{1}$ и $I_{2}$ - интервалы $(-1,1)$. Функция $h$-координата точки на первом интервале. Единственный слой на интервале $I_{2}$ совпадает с ним самим.

Эта лемма напрямую вытекает из определения и из теории Морса.

СлЕДСТВИЕ 1. Бифуркачионная диаграмма ограничения отображения момента жна $R_{\theta(x)}-$ гладкая кривая.

СлЕДСТВИЕ 2. Тангенс угла наклона касательной $к$ этой кривой на плоскости с координатами $h, f$ равен $-\lambda / \mu$, где $\lambda u \mu$ - коэффициенты зависимости между дифференциалами функиий $h$ u $f$.

Нульмерные невырожденные орбиты. Определение невырожденности нульмерных орбит принадлежит Л. М. Лерману и Я. Л. Уманскому.

Пусть в точке $x \in M^{4} d h=d f=0$. Рассмотрим систему координат $x_{1}, y_{1}$, $x_{2}, y_{2}$ с началом в ней. На пространстве $\mathfrak{I}$ симметричных матриц $4 \times 4$ введем операцию коммутирования по правилу $[A, B]=A \Omega B-B \Omega A$, где $\Omega$ - матрица симплектической формы в точке $x$, записанная в координатах $x_{1}, y_{1}, x_{2}, y_{2}, A \in \mathfrak{I}$, $B \in \mathfrak{I}$. По этой операции I преврашается в алгебру Ли. Легко проверяется, что I изоморфна $\operatorname{sp}(4, \mathbb{R})$. Пусть $H$ и $F$ - координатные записи $d^{2} f$ и $d^{2} h$. Заметим, что $H$ и $F$ коммутируют, так как $[H, F]=d^{2}\{h, f\}=0$.

Рассмотрим $\mathfrak{K}$ - коммутативную подалгебру, натянутую на $H$ и $F$.

ОПРЕДЕЛЕнИЕ. Нульмерную орбиту будем называть невырожденной, если соответствуюшая ей подалгебра $\mathfrak{K}$ - подалгебра Картана.

Корректность определения легко проверяется. Действительно, при замене координат с якобианом $I$ подалгебра $\mathfrak{K}$ переходит в сопряженную ей подалгебру $I \mathfrak{K} I^{T}$.

$\mathrm{B} \mathrm{sp}(4, \mathbb{R})$ четыре типа подалгебр Картана с точностью до сопряжения.

В соответствии с этим введем 4 типа невырожденных особых точек.

Из результатов Л. М. Лермана и Я. Л. Уманского вытекают две следующие важные леммы. Эти леммы показывают геометрический смысл типов особых точек.

Лемма 3. Топологическое строение в мальх окрестностях точек одного типа одинаково. (Для любых двух точек одного типа существует сохраняющий расслоение Лиувилля гомеоморфизм между их некоторыми малыми иаровыми окрестностями.) 
ЛЕмма 4. Любая одномерная орбита, имеющая в своем замыкании невырожденную точку ранга 0 , невырожденна.

Из леммы 3 вытекает возможность классификации невырожденных точек ранга нуль по топологическому строению в их малых окрестностях.

ОпРЕДЕЛЕНИЕ. Будем говорить, что невырожденная точка имеет тип

1) фокус-фокус, если ее некоторая окрестность топологически эквивалентна $F=\left\{D^{4}, h, f\right\}$, где $D^{4}=\left\{x_{1}^{2}+y_{1}^{2}+x_{2}^{2}+y_{2}^{2}<1\right\}$ - четырехмерньй диск, $h=x_{1} y_{1}+x_{2} y_{2}$ и $f=x_{1} y_{2}-x_{2} y_{1}-$ функции, задающие слоение;

2) седло-седло, если ее некоторая окрестность топологически эквивалентна $S=\left\{D^{4}, h, f\right\}$, где $D^{4}=\left\{x_{1}^{2}+y_{1}^{2}+x_{2}^{2}+y_{2}^{2}<1\right\}$ - четырехмерный диск, $h=x_{1} y_{1}$ и $f=x_{2} y_{2}$;

3) иентр-седло, если ее некоторая окрестность топологически эквивалентна $C S=\left\{D^{4}, h, f\right\}$, где $D^{4}=\left\{x_{1}^{2}+y_{1}^{2}+x_{2}^{2}+y_{2}^{2}<1\right\}$ - четырехмерный диск, $h=x_{1}^{2}+y_{1}^{2}$ и $f=x_{2} y_{2}$;

4) иентр-иентр, если ее некоторая окрестность топологически эквивалентна $C=\left\{D^{4}, h, f\right\}$, где $D^{4}=\left\{x_{1}^{2}+y_{1}^{2}+x_{2}^{2}+y_{2}^{2}<1\right\}$ - четырехмерньй диск, $h=x_{1}^{2}+y_{1}^{2}$ и $f=x_{2}^{2}+y_{2}^{2}$.

Топологическое строение окрестности точки типа седло-седло удобно представлять себе как прямое произведение $\left\{D^{2}, h\right\} \times\left\{D^{2}, h\right\}$, где $D^{2}=\{|x|<1,|y|<1\}$, $h=x y$.

ЛЕмма 5 (о строении куска орбиты). Пусть $W$ - связное замкнутое многообразие с краем, иеликом содержащееся в некоторой двумерной орбите. Тогда существует естественное влоэсение $W \times D^{2}, D^{2}=\left\{(f-f(x))^{2}+\right.$ $\left.(h-h(x))^{2}<\delta\right\}, f$ и щее слои функций $f$ и $h$ и тохсдественно отображсающее слой $\{f=f(x)$, $h=h(x)\}{ }_{\boldsymbol{\theta}} W$.

Действительно, рассмотрим нормальное расслоение к $W$. Пересечение его с множеством, задаваемьм неравенством $\left.(f-f(x))^{2}+h-h(x)\right)^{2}<\delta$, и является искомым вложением.

СлЕДСТВИЕ 1. Пусть пересечение $\widehat{K}$ особого слоя $K$ с некоторой окрестностью гомеоморфно прямому произведению креста (крестом мы будем называть пару пересекающихся интервалов) на интервал, причем произведению иентра этого креста на интервал соответствует невыро сденная седловая орбита. Тогда существует регулярная окрестность $\widehat{K}$, топологически әквивалентная четырехмерному диску $\left\{\left|p_{1}\right|<1,\left|q_{1}\right|<1,\left|p_{2} q_{2}\right|<1\right.$, $\left.\left|p_{2}\right|<1,\left|q_{2}\right|<1\right\}$, расслоенному на связные компоненты совместных поверхностей уровня функиий $f=p_{2} q_{2}$ u $h=p_{1}$.

\section{§3. Полный топологический инвариант седловых окрестностей}

Основная задача этого параграфа состоит в построении удобного полного топологического инварианта седловых окрестностей. Оно проводится в леммах $3,4,5$. Первые две леммы нужны для описания общих свойств седловых окрестностей. 
Под седловой окрестностью можно понимать интегрируемую гамильтонову систему $\left(U^{4}, \omega, h, f\right)$,

1) не содержашую вырожденных точек,

2) бифуркационная диаграмма которой состоит из пары трансверсально пересекающихся по точке $A$ интервалов, каждый из которых является графиком гладкой функции от $h$,

3) прообраз точки $A$ связен и содержит хотя бы одну точку типа седло-седло, $U^{4}$ есть регулярная окрестность $\varkappa^{-1}(A)$.

Под $(+)$ седловой окрестностью мы будем понимать интегрируемую гамильтонову систему $\left(U_{+}^{4}, \omega_{+}, h_{+}, f_{+}\right)$,

1) не содержашую вырожденных точек,

2) бифуркационная диаграмма которой состоит из пары трансверсально пересекающихся по точке $A$ интервалов, один из которых вертикален, а другой горизонтален,

3) прообраз точки $A$ связен и содержит хотя бы одну точку типа седло-седло, $U^{4}$ есть регулярная окрестность $\varkappa^{-1}(A)$.

Две $(+)$ седловые окрестности мы будем назьвать топологически эквивалентными, если между ними существует гомеоморфизм, сохраняющий слоение Лиувилля и ориентацию критических окружностей векторным полем $\operatorname{sgrad}_{f}+\operatorname{sgrad}_{h}$.

Докажем, что задачи классификации седловых и (+) седловых окрестностей с точностью до топологической эквивалентности эквивалентны. Действительно, пусть бифуркационная диаграмма седловой окрестности $\mathfrak{U}_{S}$ состоит из пересекающихся по точке $A$ кривых $\gamma$ и $\varphi$. Рассмотрим диффеоморфизм $\alpha:(h, f) \rightarrow\left(h_{+}, f_{+}\right)$ такой, что

1) $h_{+}=0$,

2) $\left.f_{+}\right|_{\varphi}=0$

3) $\left.h_{+}\right|_{\varphi}=h-h(A)$,

4) $\left.f_{+}\right|_{\gamma}=f-f(A)$.

Сушествование такого диффеоморфизма не вызывает сомнений. Четверка $\left(U^{4}, \omega, h_{+}, f_{+}\right)$является $(+)$седловой окрестностью. Тождественное отображение id: $U^{4} \rightarrow U^{4}$ сохраняет слоение Лиувилля и на критических окружностях переводит векторное поле $\operatorname{sgrad}_{h}$ в векторное поле $\operatorname{sgrad}_{f}+\operatorname{sgrad}_{h}$. В одну сторону доказано, в другую сторону достаточно заменить функцию $h$ на функцию $h-f$.

Остальная часть этого параграфа посвящена топологической классификации (+) седловых окрестностей.

Обозначим через $K$ прообраз $\varkappa^{-1}(A) . K$ состоит из объединения двумерных, одномерных и нульмерных орбит.

Лемма 1. Все двумернъе орбить, содержсащие в замъкании точку типа седло-седло, гомеоморфныц $\mathbb{R}^{2}$.

ДокАЗАТЕЛЬСТво. Пусть орбита $\theta^{2}$ содержит в своем замькании точку типа седло-седло. Следовательно, $\theta^{2}$ содержит в своем замыкании по крайней мере две одномерные орбиты, гомеоморфные $\mathbb{R}, \mathrm{c}$ разными коэффициентами зависимости между дифференциалами функций $f$ и $h$. Это следует из определения точки типа 
седло-седло. Для доказательства леммы нам потребуется следуюшее утверждение.

УТВЕРЖДЕНИЕ. Пусть орбита $V$ содержит в своем замыкании орбиту $U$. Тогда стабилизатор орбить $V$ содержится в стабилизаторе орбиты $U$. $\left(\bar{V} \subseteq U \Longrightarrow \mathrm{St}_{V} \supseteq \mathrm{St}_{U}.\right)$

ДокаЗАТеЛЬство. Пусть $(a, b) x=x$. Рассмотрим последовательность $x_{i}$ точек из $V$, сходящуюся к некоторой точке $y ;(a, b) x_{i}=x_{i}$. По теореме о непрерывной зависимости решения дифференциального уравнения от начальных данных $(a, b) y=y$, что и требовалось доказать.

Стабилизатор одномерной $\mathbb{R}$-орбиты есть прямая $\lambda x+\mu y=0$, где $\lambda$ и $\mu$-коэффициенты зависимости между $d f$ и $d h$ на орбите. Следовательно, стабилизатор орбиты $\theta^{2}$ содержится в пересечении несовпадаюших прямых, и, следовательно, он тривиален.

Лемма 2. Слой $K$ гомеоморфен двумерному клеточному комплексу. Двумерные грани әтого комплекса являются двумерныци орбитами, одномерньее грани (ребра) - одномерными орбитами и нульмерные грани (вершинь) - нульмерными орбитами.

ЗАмЕчАниЕ. В следующем параграфе будет показано, что двумерные грани четырехугольники, т.е. двумерную грань ограничивают ровно четыре ребра.

Сначала докажем следующее утверждение.

УТВЕРЖДЕНИЕ. Пусть $\theta^{1}$ - одномерная орбита, имеющая в замыкании невырожденные точки ранга нуль $x_{1}$ и $x_{2}$, причем точка $x_{1}$ имеет тип седло-седло. Тогда точка $x_{2}$ тоже имеет тип седло-седло.

ДоКАЗАТЕЛЬСТВО этого утверждения почти тривиально и основывается на простом переборе. Всего есть четыре типа невырожденных точек ранга 0. Точки типа центр-центр и фокус-фокус не могут лежать в замькании одномерной орбиты. Все одномерные орбиты, в замыкании которых лежит точка типа центр-седло, центральные. Все одномерные орбиты, в замыкании которых лежит точка типа седло-седло, седловые, из чего и следует утверждение.

ДоКАЗАТЕЛЬСТво ЛЕмМЫ 2 следует из леммы 1 , строения $K$ в окрестности особых точек и из этого утверждения. Пусть $C=\left\{x \in \mathfrak{U}_{S}: \operatorname{rank}(x)<2\right\}$ - множество особых точек. Рассмотрим $L-$ связную компоненту множества $C \cap K$, содержашую точку типа седло-седло. Легко видеть, что $L$ - граф̆, ребрами которого являются седловые орбиты, а вершинами невырожденные точки типа седло-седло. Рассмотрим $\mathscr{O}$ - множество всех двумерных орбит, имеющих в замыкании хотя бы одну точку из $L$. Докажем, что все такие орбиты гомеоморфны $\mathbb{R}^{2}$. Действительно, из теоремы о непрерывной зависимости решения дифференциального уравнения от начальных условий следует, что если замыкание орбиты содержит в себе некоторую точку другой орбиты, то оно содержит в себе всю другую орбиту вместе с ее замыканием. Поэтому орбиты из этого множества содержат в своем замыкании точку типа седло-седло и по лемме 1 гомеоморфны $\mathbb{R}^{2}$. 
Из связности $K$ следует, что слой $K$ является объединением всех орбит из $\mathscr{O}$ и графа $L, K=\bigcup_{\theta \in \mathscr{O}} \theta \cup L$, т.е. клеточным комплексом с клетками описанного вида. Лемма доказана.

Можно считать, что бифуркационная диаграмма седловой окрестности состоит из объединения двух интервалов $\{x=0,|y|<1\}$ и $\{y=0,|x|<1\}$, так как при диффеоморфизме образа отображения момента система переходит в топологически эквивалентную и невырожденность точек и типа точек ранга нуль сохраняются. Из следствия 2 леммы $2 \S 2$ это условие эквивалентно следуюшему: $(\lambda d f+\mu d h=0$, $\lambda \mu=0)$.

Граф $L$ является одномерным остовом комплекса $K$. Граф $L-$ связный граф степени 8. Ребра его естественно разбиваются на два класса: $h$-класс, задаваемый условиями $d h \neq 0, d f=0$; и $f$-класс, задаваемый условиями $d f \neq 0, d h=0$. На ребрах введем ориентацию векторньм полем $\operatorname{sgrad}_{f}+\operatorname{sgrad}_{h} . h$-граф и $f$-граф̆ имеют степень четыре; в каждую вершину $L$ входят два $h$-ребра и два $f$-ребра, из каждой вершины $L$ выходят два $h$-ребра и два $f$-ребра. Окрестности всех вершин графа $L$ идентичны. В каждую вершину входят два $f$ и два $h$-ребра и выходят два $f$ и два $h$-ребра. Соответственно, окрестность каждой вершины состоит из двух входящих и двух выходящих $f$-лучей и двух входящих и двух выходящих $h$-лучей. Такие вершины будем называть стандартными .

В дальнейшем под одномерныцм остовом мы будем понимать ориентированньй, разбитьй на $f$ - и $h$-подграфы одномерный остов особого слоя седловой окрестности. Примеры одномерных остовов изображены на рис. 2.

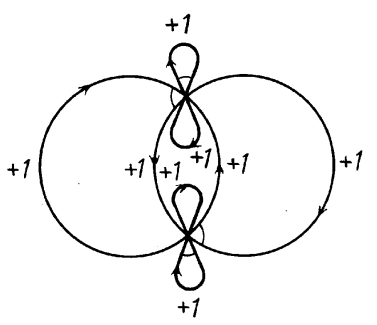

Рис. 2a

Будем говорить, что $(+)$ седловая окрестность $U$ тоньше, чем седловая окрестность $V$, если $\bar{U} \subseteq V$. Очевидно, что все $(+)$ седловые окрестности одного слоя $K$ топологически эквивалентны. В дальнейшем мы подразумеваем, что $(+)$ седловая окрестность достаточно тонкая.

ЛЕмма 3 (о разбиении; основная лемма этого параграфа). Пусть $\mathfrak{U}$ - седловая окрестность. Тогда ее можсно разрезать на $m_{0}+m_{2}$ кусков, $m_{0}$ из которых топологически әквивалентны $S^{4}=\left\{S^{2}, g\right\} \times\left\{S^{2}, g\right\}$, и $m_{2}$ из которых топологически әквивалентны $D^{2} \times \mathbb{R}^{2}$, где: $S^{2}$ - двумерный диск $\{|x|<1,|y|<1$, $|x y|<1 / 2\}, g=x y, D^{2}$ - двумерный диск с единственным слоем, совпадающим с ним самим, $\mathbb{R}^{2}$ тривиально расслоена на точки. $m_{0}$ - число вершин, $m_{2}$ - число двумерных граней в комплексе $K$. 


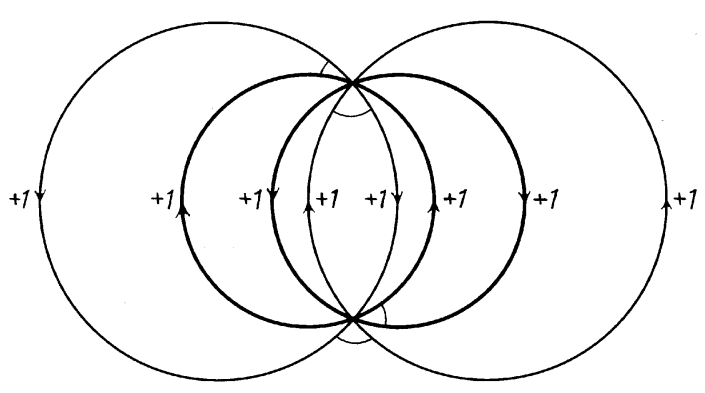

Рис. 2 бб

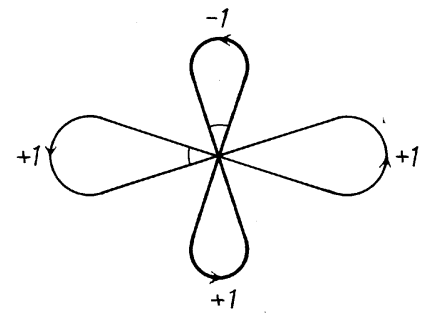

Рис. 2в

Подразумевается, что функция $g$ задает слоение на диске $S^{2}$. Напоминаем, что слоями на прямом произведении здесь и далее считаем всевозможные прямые произведения слоев на компонентах.

ДокАЗАТЕльство. Для каждой точки типа седло-седло $p_{i}$ выберем окрестность $V_{i}$, топологически эквивалентную $S^{4}=\left\{S^{2}, g\right\} \times\left\{S^{2}, g\right\}$. Сушествование такой окрестности следует из определения точки типа седло-седло. Окрестность $\mathfrak{U}$ считаем такой тонкой, что образ $\varkappa(\mathfrak{U}) \subset \bigcap_{i} \varkappa\left(V_{i}\right)$. Заметим, что $\mathfrak{U} \cap V_{i}$ также топологически эквивалентны $S=\left\{S^{2}, g\right\} \times\left\{S^{2}, g\right\}$.

Для каждой двумерной грани $Y_{i}$ выделим ее пересечения со всеми $V_{i}$. Это пересечение либо пусто, либо является некоторыми окрестностями-уголками вершин $Y_{i}$. Соединим вершины соседних уголков. Полученные отрезки ограничивают многоугольник $\widehat{Y}_{i}$. По лемме $5 \oint 2$ существует окрестность $W_{i}$ многоугольника $\widehat{Y}_{i}$, топологически эквивалентная $D^{2} \times \mathbb{R}^{2}$, слоем на $\mathbb{R}^{2}$ считаем точку. Считаем окрестность $\mathfrak{U}$ такой тонкой, что $\varkappa(\mathfrak{U}) \subseteq \bigcap_{i} \varkappa\left(V_{i}\right) \bigcap_{j} \varkappa\left(W_{j}\right)$. Можно считать, что двумерный участок гранищы $V_{i}$, трансверсальный слоям и проходящий через вершину $\widehat{Y}_{i}$, совпадает с двумерным трансверсальным слоем и проходящим через ту же вершину многоугольника $\widehat{Y}_{i}$ участком границы $W_{i}$.

Рассмотрим разность $\mathfrak{G}=\mathfrak{U} \backslash\left(\bigcup_{i} V_{i} \bigcup_{j} W_{j}\right)$. Она состоит из трубчатых 
окрестностей ребер комплекса $K$. Если окрестность $\mathfrak{U}$ достаточно тонкая, то по следствию из леммы $5 \S 2$ каждая такая окрестность топологически эквивалентна $T=\left\{S^{2}, g\right\} \times\left\{I_{1}, t\right\} \times I_{2}$. Присоединение их к одному из граничащих с каждой из них $V_{i}$ не меняет топологическую структуру последнего.

Мы получили разбиение $\mathfrak{U}$ на $m_{0}+m_{2}$ кусков, $m_{0}$ из которых эквивалентны $S$, и $m_{2}$ из которых эквивалентны $D^{2} \times \mathbb{R}^{2}$. Лемма доказана.

ЗАмЕчАнИЕ 1. Так как любые две седловые окрестности одного слоя топологически эквивалентны, то разбиение тонкой окрестности $\widehat{\mathfrak{U}}$ порождает эквивалентное разбиение “толстой” окрестности $\mathfrak{U} \supseteq \widehat{\mathfrak{U}}$.

Описанное разбиение будем называть базовым.

ЗАмЕчАниЕ 2. Любые два разбиения, удовлетворяющие перечисленным в лемме 3 свойствам, в некотором смысле "очень похожи". Фиксируем для каждой седловой окрестности $\mathfrak{U}$ некоторое базовое разбиение.

ОПРЕДЕЛЕНИЕ. Толстым скелетом седловой окрестности мы будем называть объединение всех кусков типа $S$ (вместе с попарно общей для двух кусков границей).

Толстый скелет является регулярной окрестностью графа $L$.

Следующая лемма показьвает, что вся информация о топологическом строении седловой окрестности заключена в ее толстом скелете.

ЛЕмма 4. Пусть $\mathfrak{U}$ и $\widehat{\mathfrak{U}}-$ две седловие окрестности, $\mathfrak{G}$ и $\widehat{\mathfrak{G}}-$ их толстые скелеты. Тогда $\mathfrak{U}$ топологически әквивалентна $\widehat{\mathfrak{U}}$, если и только если $\mathfrak{G}$ топологически эквивалентен $\widehat{\mathfrak{G}}$.

ДокАЗАТЕльство. В одну сторону лемма очевидна. Докажем в другую сторону. Пусть $\mathfrak{G}$ и $\widehat{\mathfrak{G}}$ топологически эквивалентны.

УТВЕРЖ ДЕНИЕ 1. Пусть $P^{3}=S^{1} \times D^{2}-$ тривиально расслоенное на окружности полноторие. ( $S^{1}$ - окружность, единственный слой на ней совпадает с ней самой, $D^{2}$ - двумерный диск, тривиально расслоенный на точки.) Тог-

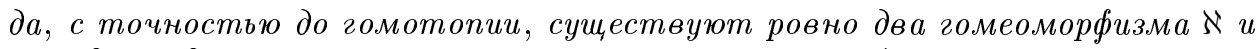
$\aleph^{\prime}: P^{3} \rightarrow P^{3}$, сохраняющих слоение. Если ввести на $S^{1}$ ориентацию, то одно из этих отображений ( $)$ сохраняет ориентацию слоев, а другое $\left(\aleph^{\prime}\right)$ меняет ориентацию на противополохную.

Это утверждение достаточно известно, и мы не будем его здесь доказывать.

Разрежем $\mathfrak{U}$ на $\mathfrak{G}$ и $W_{i}$. Многообразиями разреза являются $m_{2}$ тривиально расслоенных на окружности полноторий $P_{i}^{3}$. Полноторие $P_{i}^{3}$ является обшей границей $W_{i}$ и $\mathfrak{G}$.

УТВЕРЖДЕНИЕ 2. Пусть $W=\bar{D}^{2} \times \mathbb{R}^{2}$, слоение на $\mathbb{R}^{2}$ тривиально, единственный слой на диске $\bar{D}^{2}$ совпадает с ним самим, $P^{3}=\partial D^{2} \times \mathbb{R}^{2}$ - граничное полноторие, гомеоморфизмы $\aleph \aleph^{\prime}: P^{3} \rightarrow P^{3}$ негомотопны и сохраняют слоение. Тогда существует такой сохраняющий слоение гомеоморфизм $\varphi: W \rightarrow W$, чmо $\varphi(\aleph)=\aleph^{\prime}$. 
ДокАЗАТЕЛЬСТво. В качестве такого гомеоморфизма можно взять, например, гомеоморфизм, порождаемый симметрией диска $\bar{D}^{2}$ относительно его диаметра. Легко проверяется, что он удовлетворяет условиям леммы.

Седловые окрестности $\mathfrak{U}$ и $\widehat{\mathfrak{U}}$ получаются из толстых скелетов $\mathfrak{G}$ и $\widehat{\mathfrak{G}}$ приклеиванием "шапок" $W_{i}$ и $\widehat{W}_{i}$ по граничным полноториям. Утверждения 1 и 2 показывают, что соответствующие склеивающие отображения можно выбрать так, что они станут гомотопными. Любые две "шапки" топологически эквивалентны, и два любых слоя шапки гомеоморфны. Из гомотопности склейки границ следует гомотопность склейки слоев, следовательно, существует гомеоморфизм $\varkappa: \mathfrak{U} \rightarrow \widehat{\mathfrak{U}}$, сохраняюший слоение, что и требовалось доказать.

ОПРЕДЕЛЕНИЕ. Толстый скелет $\mathfrak{G}$ назовем допустимым, если существует седловая окрестность $\mathfrak{U}$ с толстьм скелетом $\mathfrak{G}$.

Задача построения полного топологического инварианта для седловых окрестностей сведена к задаче построения полного топологического инварианта для допустимых толстых скелетов.

Рассмотрим поподробнее "куски" $V_{i}$, из которых склеен толстый скелет $\mathfrak{G} ; V_{i}$ топологически эквивалентны $S=\left\{S_{1}^{2}, h\right\} \times\left\{S_{2}^{2}, f\right\}$, где

$S_{1}^{2}$ - двумерньй диск $\left\{\left|x_{1}\right|<1,\left|y_{1}\right|<1,\left|x_{1} y_{1}\right|<1 / 2\right\}, h=x_{1} y_{1}$,

$S_{2}^{2}$ - двумерньй диск $\left\{\left|x_{2}\right|<1,\left|y_{2}\right|<1,\left|x_{2} y_{2}\right|<1 / 2\right\}, f=x_{2} y_{2}$ (рис. 3 ).

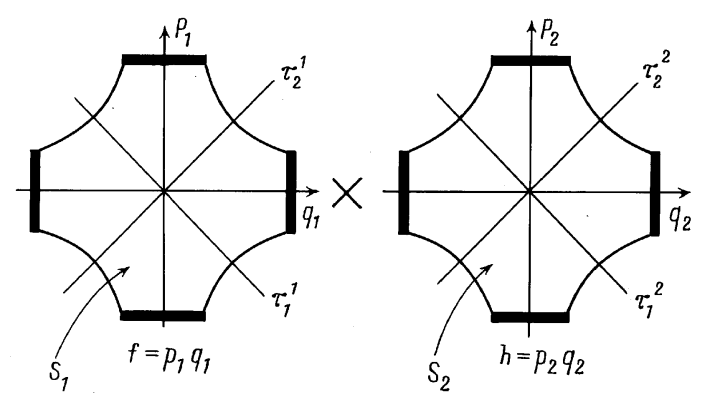

Рис. 3

Подмногообразие $B_{i}$ границ $\partial V_{i}$, по которому толстый скелет $\mathfrak{G}$ разрезается на $V_{i}$, состоит из объединения трехмерных дисков

$$
F_{i}^{a} \sim a_{h} \times S_{2}^{2}, \quad F_{i}^{a} \sim a_{h}^{\prime} \times S_{2}^{2}, \quad H_{i}^{a \sim} S_{1}^{2} \times a_{f}, \quad H_{i}^{a} \sim S_{1}^{2} \times a_{f}^{\prime}
$$

Значок говорит в данном случае о существовании гомоморфизма, сохраняюшего функции $h$ и $f$. Отметим, что эти диски не пересекаются.

На каждом таком диске рассмотрим слоение, задаваемое отображением момента $\varkappa$. 
Особым слоем назовем слой, содержащий хотя бы одну точку, ранг дифференциала отображения момента в которой менше двух. Легко видеть, что все особые слои гомеоморфны паре пересекающихся интервалов (кресту). Любой неособый слой гомеоморфен интервалу. Объединение всех неособых слоев топологически эквивалентно прямому произведению креста на интервал, слоем на интервале считаем любую точку.

На особых слоях введем ориентацию. Ориентацией особого слоя назовем задание направлений на каждом из четырех интервалов, получаемых из креста выкидыванием центральной точки. Рассмотрим особый слой, соответствующий пересечению комплекса $K$ с граничным диском. $V_{i}$ высекает на $K$ фигуру, гомеоморфную прямому произведению креста на крест. Кресты, являющиеся прямым произведением вершины одного креста на другой крест, ориентируем векторным полем sgrad $f+\operatorname{sgrad}_{h}$ (их объединение является окрестностью вершины графа $L$ ). Ориентируем кресты - компоненты прямого произведения - так, чтобы полученная ориентация на окрестностиграфа $L$ совпа дала с действительной. Заметим, что это можно сделать единственным образом. Ориентация компонент прямого произведения порождает ориентацию граничных крестов, и, следовательно, одного особого слоя на граничном диске. Ориентация остальных особых слоев на граничном диске определяется аналогичньм способом.

Лемма 5. Пусть $B^{3}=\left\{S^{2}, h\right\} \times\{I, f\}$, әде, как и прежсде, $S^{2}-$ двумерный диск $\{|x|<1,|y|<1,|x y|<1 / 2\}, h=x y$, функиия $f$ расслаивает интервал I на точки. Пусть на особъх слоях $B^{3}$ задана ориентация. Тогда, с точностью до изотопии, сохраняющей слои, существуют ровно два гомеоморфизма $\xi: B^{3} \rightarrow B^{3}$ maкux, чmо:

1) $\xi$ сохраняет слоение Лиувилля;

2) $\xi$ сохраняет знак функиий $h u f$, m.e. $\operatorname{sgn}(\xi(h))=\operatorname{sgn}(h), \operatorname{sgn}(\xi(f))=$ $\operatorname{sgn}(f)$

3) в сохраняет ориентацию особых слоев.

Сначала докажем, что по крайней мере два таких гомеоморфизма сушествуют. Рассмотрим симметрию $S^{2}$ относительно ее центральной точки 0. Гомеоморфизм, порожденный этой симметрией, удовлетворяет условиям леммы. Докажем, что он не изотопен тождественному. Рассмотрим крест $\{h=0, f=0\}$. Так как изотопия сохраняет знаки функций, то она оставляет этот крест на месте, не переворачивая его. С другой стороны, симметрия переворачивает этот крест.

Выделим два входящих в точку 0 луча $\alpha$ и $\beta$ креста $\{h=0, f=0\}$. Докажем, что если $\xi(\alpha)=\alpha, \xi(\beta)=\beta$, то гомеоморфизм $\xi$ изотопен тождественному.

УТВЕРЖДЕНИЕ 3. Пусть $z$ - топологическое пространство, I - отрезок. Обозначим через $Z$ прямое произведение $z \times I$. Рассмотрим множество $P$ всех гомеоморфизмов из $Z$ в $Z$, переводящих произведение z на любой конеи отрезка в себя и сохраняющих тривиальное расслоение на отрезке. Будем считать два таких гомеоморфизма эквивалентными, если один можно перевести в другой изотопией, сохраняющей слоение. Тогда любой класс эквивалентности множества $P$ имеет своим представителем гомеоморфизм, порождаемый гомеоморфизмом пространства $z$. 
Это утверждение достаточно известно, и мы не будем его здесь доказывать.

Многообразие $B^{3}$ является прямым произведением $S^{2}$ на интервал $I$. Рассмотрим слой этого разбиения, содержащий крест $\{h=0, f=0\}$. На этом слое рассмотрим множество $V=\{p \geqslant 0, q \geqslant 0\}$. Это множество можно рассматривать как прямое произведение отрезка на полуинтервал (здесь удобнее считать, что интервалы $\{|p|=1\},\{|q|=1\}$ входят в $S^{2}$ ). Ориентация особых слоев индуцирует ориентацию всех слоев на $V$, из сохранения ее вытекает сохранение произведений концевых точек на интервал. Используя утверждение, получаем, что все гомеоморфизмы $\pi: S^{2} \rightarrow S^{2}$, сохраняющие слои, изотопны тождественному. Для доказательства леммы достаточно еще раз применить утверждение.

Построение инварианта. Подытожим. Из лемм 3,4 вытекает, что топологическое строение седловой окрестности может быть задано склейкой одинаковых кусков $V_{i}$ по некоторым участкам их границ. Из леммы 5 вытекает, что для каждой пары таких участков с топологической точки зрения существует только два гомеоморфизма склейки. Следовательно, для того, чтобы задать $\mathfrak{U}_{S}$ с точностью до топологической эквивалентности, достаточно указать склеивающиеся участки и выбрать один из двух возможных гомеоморфизмов склейки.

Пусть $\mathfrak{U}_{S}$ - седловая окрестность, $L$ - одномерный остов особого слоя $K$. Рассмотрим базовое разбиение окрестности $\mathfrak{U}_{S}$. Это разбиение индуцирует разбиение остова $L$ на куски $L_{i}$, каждый из которых стандартен, т.е. состоит из двух входящих и двух выходящих $f$-лучей и двух входящих и двух выходящих $h$-лучей. Перенумеруем вершины графа $L$ в соответствии с номерами $V_{i}$. Для каждого $i$ фиксируем сохраняющий слоение гомеоморфизм $\pi_{i}$ между $V_{i}$ и $S^{4}=\left\{S_{1}^{2}, h\right\} \times\left\{S_{2}^{2}, f\right\}$. На дисках $S_{1}^{2}$ и $S_{2}^{2}$ выделим пару лучей особого креста каждого диска как на рис. 4 . Гомеоморфизм $\pi_{i}$ индуцирует выделение пары лучей каждого класса на $L_{i}$, на рис. 4 и далее выделенная пара лучей будет соединяться дужкой.

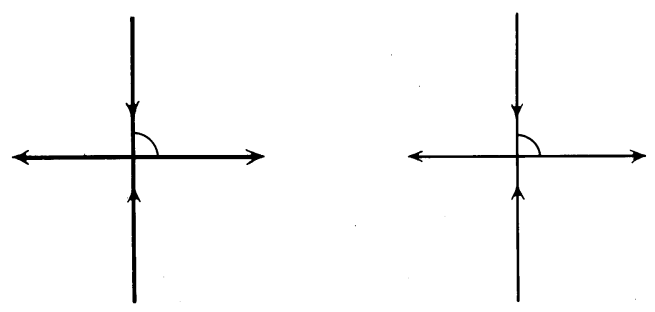

Рис. 4

УТВЕРЖДЕНИЕ 4. Склейка $L_{i}$ в $L$ однозначно задает склеивающиеся участки границ, кусков $V_{i}$.

Это утверждение очевидно.

Заметим, что описанное выше выделение пар лучей позволяет задавать одну из двух топологически различных склеек склеивающихся участков границ. Действительно, введем ориентации на особых слоях склеивающихся участков границ. 
Выделим на каждом таком особом слое-кресте пару лучей этого креста, один из которых входит, а второй выходит из вершины креста. Удовлетворяющий условиям леммы 5 гомеоморфизм переводит выделенную пару лучей в выделенную пару или выделенную пару в пару, ни один луч которой не входит в выделенную пару. В первом случае на ребре графа $L$, соответствуюшем склейке, ставим метку $\varepsilon=1$, во втором - метку $\varepsilon=(-1)$.

ОПРЕДЕЛЕНИЕ. Оснащением остова $L$ назовем

1) выделение для каждой вершины по паре ребер, одно из которых выходит, а другое входит в эту вершину, каждого класса,

2) постановка в соответствие каждому ребру метки $\varepsilon \in\{1,-1\}$ в соответствии с описанными выше правилами.

Из всего вышеописанного вытекает следуюшее утверждение.

УТВЕРЖДЕНИЕ 5. Полученный граф с оснащением однозначно задает топологическое строение седловой окрестности $\mathfrak{U}_{S}$.

Реальные примеры оснащенных остовов изображены на рис. 2.

Заметим, что по седловой окрестности $\mathfrak{U}_{S}$ могут быть построены разные оснащенные остовы. Введем отношение эквивалентности на множестве оснашенных остовов так, чтобы все оснашенные остовы, соответствующие одной седловой окрестности, содержались в одном классе.

Рассмотрим $\mathfrak{L}_{n}-$ множество ориентируемых графов

a) с $n$ вершинами,

b) степени 8 ,

c) ребра которых разбиты на два класса (черные и белые ребра),

d) в каждую вершину входят и выходят по два ребра каждого класса,

е) для каждой вершины пара ребер каждого класса, одно из которых входяшее в вершину, а другое выходяшее, выделена,

f) на каждом ребре стоит метка $\varepsilon \in\{1,-1\}$.

Введем на множестве $\mathfrak{L}_{n}$ отношение эквивалентности, порождаемое следующими операциями.

( ) Для любой вершины графа $L$ выделим невыделенную пару белых ребер, а прежде выделенную пару считаем невыделенной. При этом метки $\varepsilon$ на каждом выходящем или входяшем в эту вершину черном ребре, не являюшемся петлей, умножаем на $(-1)$.

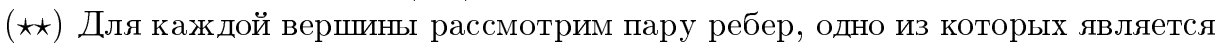
выделенным выходящим, а другое невыделенньм входящим. Будем считать выделенной эту пару.

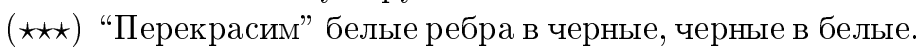

ЗАмечАниЕ. Легко видно, что два любых эквивалентных графа задают топологически эквивалентные седловые окрестности.

ОПРЕДЕЛЕНИЕ. Класс эквивалентности множества $\mathfrak{L}_{n}$ назовем CL-типом. Описанное выше отображение из множества всех седловых окрестностей в множество всех CL-типов назовем CL-отображением. 
CL-тип однозначно задает топологическое строение $\mathfrak{U}_{S}$. Чтобы определение CL-отображения было корректно, необходимо, чтобы по седловой окрестности $\mathfrak{U}_{S}$ однозначно строился $\mathrm{CL}-\mathrm{тип} \mathrm{CL}\left(\mathfrak{U}_{S}\right)$.

ЛЕмма 6. Определение CL-отображсения корректно.

ДокаЗАтЕЛЬство. Рассмотрим геометрическую интерпретацию CL-типа. Рассмотрим множества

$$
B_{1}=\left\{x \in \mathfrak{U}_{S}:\left.\operatorname{rank} d h\right|_{x}=0\right\}, \quad B_{2}=\left\{x \in \mathfrak{U}_{S}:\left.\operatorname{rank} d f\right|_{x}=0\right\}
$$

Легко видеть, что множества $B_{i}$ - двумерные многообразия, функции $f$ на $B_{1}$ и $h$ на $B_{2}$ имеют морсовскую седловую особенность в точках типа седло-седло. Следовательно, $\left(B_{1}, f\right)$ и $\left(B_{2}, h\right)$ можно рассматривать как атомы. Особые слои этих атомов являются, соответственно, $f$ - и $h$-подграфами одномерного остова $L$.

ОПРЕДЕЛЕНИЕ. $L$-типом назовем пару атомов $\left[\left(B_{1}, f\right),\left(B_{2}, h\right)\right]$.

ОПРЕДЕЛЕНИЕ. Геометрическим CL-типом седловой окрестности назовем четверку $\left\{K,\left[\left(B_{1}, f\right),\left(B_{2}, h\right)\right], \zeta_{1}, \zeta_{2}\right\}$, где двумерный комплекс $K$ - особый слой $\mathfrak{U}_{S},\left[\left(B_{1}, f\right),\left(B_{2}, h\right)\right]-L$-тип $\mathfrak{U}_{S}, \zeta_{1}$ и $\zeta_{2}$ - естественные вложения особых слоев букв $\left(B_{1}, f\right)$ и $\left(B_{2}, h\right)$ в одномерный остов комплекса.

Предполагаем, что два геометрических CL-типа эквивалентны, если их соответствующие компоненты эквивалентны. Эквивалентность на множестве всех двумерных комплексов определим как гомеоморфизм. Эквивалентность двух букв означает сохраняющий слоение гомеоморфизм между ними. Два вложения эквивалентны тогда и только тогда, когда они гомотопны, причем можно устроить гомеоморфизмы и гомотопии так, что соответствующие диаграммы будут коммутативны.

Очевидно, что по седловой окрестности однозначно строится геометрический CL-тип.

Легко проверяется следующее утверждение.

УТВЕРЖДЕНИЕ 6. По геометрическому CL-типу однозначно восстанавливается CL-mun.

Это утверждение доказывает лемму.

Утверждение 5 и лемму 6 скомпануем в следующую теорему.

ТЕОРемА 1. CL-тип - полный топологический инвариант седловой окрестности.

Напоминаем, что под полнылм инвариантом некоторого множества $A$ понимается инъективное отображение этого множества в другое множество. Полньй инвариант называют реализуемьм, если это отображение - биекция.

Эта теорема решает проблему распознавания седловых окрестностей. Действительно, для проверки, являются ли две седловые окрестности топологически эквивалентными, достаточно построить и сравнить CL-типы соответствующих им $(+)$ седловых окрестностей. 


\section{§4. Реализуемый топологический инвариант седловых окрестностей}

CL-тип не является реализуемым топологическим инвариантом.

Реализацией CL-типа $\mathbf{L}$ назовем такую седловую окрестность $\mathfrak{U}_{S}$, что $\mathrm{CL}\left(\mathfrak{U}_{S}\right)=\mathbf{L}$.

Легко построить пример нереализуемого CL-типа. Следуюшая лемма проясняет вопрос реализуемости для CL-типа.

Лемма 1. Пусть комплекс $K$ является особым слоем седловой окрестности. Тогда все двумерные грани комплекса $K$ - четырехугольники.

Эта лемма доказана в [5]. Мы приведем другое доказательство.

ДоказАТеЛЬство. Рассмотрим замыкание двумерной грани $\theta$. Это многоугольник, сторонами которого являются невырожденные одномерные орбиты, а вершинами - точки типа седло-седло. Очевидно, что он четноугольник, $h$ - и $f$-стороны которого чередуются. Рассмотрим точку $x \in \theta$. Через $\sigma(x)$ будем обозначать интегральную траекторию векторного поля $\operatorname{sgrad}_{h}$, проходящую через точку $x$. Из компактности следует, что $\overline{\sigma(x)} \backslash \sigma(x)$ непусто. Пусть точка $w \in(\overline{\sigma(x)} \backslash \sigma(x))$. Очевидно, что $w \in \bar{\theta} \backslash \theta$. Рассмотрим множество $r=\{(0, b) w, b \in \mathbb{R}\}$. Напоминаем, что $(a, b) y$ обозначает точку, получаемую из точки $y$ сдвигом вдоль векторного поля $a \operatorname{sgrad}_{h}+b \operatorname{sgrad}_{f}$ на время 1. Заметим, что множество $r$ является либо $f$-орбитой, либо точкой ранга 0. Рассмотрим $\Sigma_{r}=\{\sigma: \sigma \cap r \neq \varnothing\}-$ множество всех интегральных траекторий векторного поля $\operatorname{sgrad}_{h}$, выходящих из или входящих в множество $r$, и множество $\Sigma$ всех интегральных траекторий $\operatorname{sgrad}_{h}$. Легко видеть, что $\Sigma=\Sigma_{r}$. Действительно, пусть точка $x \in \theta, \sigma(x) \in \Sigma_{r}, y=(a, b) x$.

Рассмотрим отображение $\pi: \sigma(x) \rightarrow \theta$, определяемое по правилу $\pi(y)=(0, b) y$. Это отображение переводит $\sigma(x)$ в $\sigma(z)$, оно непрерывно и $(0, b) w \in r$. Следовательно, $\Sigma=\Sigma_{K}$.

Итак, объединение всех интегральных траекторий $\operatorname{sgrad}_{h}$, выходящих из $f$-орбиты, совпадает с $\theta$. Из явного вида функций $f$ и $h$ в окрестности невырожденной седловой $h$-орбиты, вытекает, что интегральные траектории векторного поля $\operatorname{sgrad}_{f}$ трансверсально входят или выходят из нее. Следовательно, $\theta$ ограничивают ровно две $h$-стороны, и многоугольник $\theta$ является четырехугольником.

Пусть меченый остов $L^{*}$ CL-типа $\mathbf{L}$ реализуется некоторой седловой окрестностью $\mathfrak{U}_{S}$. Рассмотрим двумерную грань $Y$ особого слоя $K$ седловой окрестности $\mathfrak{U}_{S}$. Предположим, что $Y$ приклеена к имеющим обшую вершину $A$ ребрам $a$ и $b$ одномерного остова $L$ комплекса $K$. Ребро $c$ - отличная от ребра $b$ и имеюшая с $b$ обшую вершину $B$ сторона многоугольника $Y$.

Тогда

1) ребра $a$ и $b$ принадлежат к разньм классам, $a$ и $c$ - одного класса;

2 ) если $a-$ входяшее в вершину $A$ ребро, то $c$ - входящее в вершину $B$; если $a-$ выходящее из $A$ ребро, то и $c$ - выходящее из вершины $B$;

3 ) если $а$ содержится в выделенной паре вершины $A$, а метка $\varepsilon$ ребра $b$ равна 1 , то ребро $c$ содержится в выделенной паре вершины $B$; если $a$ не содержится в выделенной паре вершины $A$, а метка $\varepsilon$ ребра $b$ равна 1 , то ребро $c$ не содержится в выделенной паре вершины $B$;

4) если ребро $a$ содержится в выделенной паре вершины $A$, а метка $\varepsilon$ ребра $b$ равна $(-1)$, то ребро $c$ не содержится в выделенной паре вершины $B$; 
если $a$ не содержится в выделенной паре вершины $A$, а метка $\varepsilon$ ребра $b$ равна $(-1)$, то ребро $c$ содержится в выделенной паре вершины $B$.

Правила 1-4 вытекают из определения CL-типа. Заметим, что для любых двух ребер остова $L^{*}$ из разных классов с обшей вершиной сушествует приклеенная к этим ребрам грань. Остальные стороны-ребра этой грани однозначно восстанавливаются правилами 1-4.

ОПРЕДЕЛЕНИЕ. Назовем правильным путем любой замкнутый путь на графе $L$, удовлетворяющий правилами 1-4. (То есть для любой тройки последовательных ребер $a, b$ и $c$ выполняются условия $1-4$.)

Из всего вьшесказанного вытекают следующие утверждения.

УТВЕРЖДЕНИЕ 1. Для любого правильного пути существует грань комплекса $K$, сторонь которой образуют этот путь.

Лемма 2. Все правильные пути реализуемого CL-типа имеют длину 4.

Эта лемма является очевидным следствием леммы 1 и утверждения 1.

ОПРЕДЕЛЕНИЕ. Конечный CL-тип $L^{*}$ будем назьвать допустимымм, если все его правильные пути имеют длину 4.

ТЕоремА 1 (реализации). Любой допустимый CL-muп реализуем.

ДокАЗАТЕЛЬСТво этой теоремы будет конструктивным. Пусть меченьй остов $L^{*}$ входит в некоторый допустимый CL-тип $\mathbf{L}$. По $L^{*}$ построим седловую окрестность $\mathfrak{U}_{S}, \mathrm{CL}$-тип которой совпадает с $\mathfrak{L}$.

ОПРЕДЕЛЕНИЕ. Блоком назовем четверку $\left\{S^{4}, \omega, f, h\right\}$, где четырехмерный диск $S^{4}=\left\{\left|p_{1} q_{1}\right|<1 / 2,\left|p_{1}\right| \leqslant 1,\left|q_{1}\right| \leqslant 1,\left|p_{2} q_{2}\right|<1 / 2,\left|p_{2}\right| \leqslant 1,\left|q_{2}\right| \leqslant 1\right\}$, 2-форма $\omega=d p_{1} \wedge d q_{1}+d p_{2} \wedge d q_{2}, f=p_{1} q_{1}, h=p_{2} q_{2}$.

Удобно представлять себе блок как прямоепроизведение $\left\{S_{1}^{2}, f, \omega_{1}\right\} \times\left\{S_{2}^{2}, h, \omega_{2}\right\}$, где двумерные диски $S_{i}^{2}=\left\{\left|p_{i} q_{i}\right|<1 / 2,\left|p_{i}\right| \leqslant 1,\left|q_{i}\right|<1\right\}, \omega_{i}=d p_{i} \wedge d q_{i}$, $f=p_{1} q_{1}, h=p_{2} q_{2}, i=1,2$. Такое представление блока в виде прямого произведения назовем моделью 2 , рис. 3 . Заметим, что функции $h$ и $f$ на блоке находятся в инволюции. Блок - интегрируемая гамильтонова система на многообразии с краем. На нем можно определить отображение момента $\varkappa$, переводяшее точку $x$ блока в пару чисел $(h(x), f(x))$.

Пусть количество вершин графа $L^{*}$ равно $n$. Возьмем $n$ блоков. Все обозначения из определения блока переносятся на $k$-й блок добавлением индекса $k$ снизу или сверху. Склеим некоторые участки границы всех блоков и введем структуру дифференцированного многообразия так, что результат склейки будет седловой окрестностью $\mathfrak{U}_{S}$ CL-типа $\mathfrak{L}$.

Чтобы задать склейку необходимо задать склеивающиеся участки границы и склеивающие отображения.

Склеивающиеся участки. Рассмотрим подмножества гранищы

$$
G_{h}^{k}=\left\{\left|p_{1}\right|=1\right\} \cup\left\{\left|q_{1}\right|=1\right\}, \quad G_{f}^{k}=\left\{\left|p_{2}\right|=1\right\} \cup\left\{\left|q_{2}\right|=1\right\} .
$$


Пересечение $G_{f}^{k}$ с $G_{h}^{k}$ непусто и равно всевозможным прямым произведениям жирно выделенных граничных отрезков на модели 2 .

Разобьем каждое из множеств $G_{h}^{k}, G_{f}^{k}$ на два подмножества следующим образом: $G_{f+}^{k}=\left\{\left|p_{1}\right|=1\right\}, G_{f-}^{k}=\left\{\left|q_{1}\right|=1\right\}, G_{h+}^{k}=\left\{\left|p_{2}\right|=1\right\}, G_{h-}^{k}=\left\{\left|q_{2}\right|=1\right\}$. Заметим, что векторное поле $\operatorname{sgrad}_{h}$ трансверсально входит в $G_{h+}^{k}$ и трансверсально выходит из $G_{h-}^{k}$. Аналогично, векторное поле $\operatorname{sgrad}_{f}$ трансверсально входит в $G_{f+}^{k}$ и трансверсально выходит из $G_{f-}^{k}$. Введем обозначения $G_{f+}=\bigcup_{k} G_{f+}^{k}$, $G_{f-}=\bigcup_{k} G_{f-}^{k}, G_{h+}=\bigcup_{k} G_{h+}^{k}, G_{h-}=\bigcup_{k} G_{h-}^{k}, G_{f}=\bigcup_{k} G_{f}^{k}, G_{h}=\bigcup_{k} G_{h}^{k}$.

На каждом блоке рассмотрим множество $L^{k}=\{f=0, h=0, \operatorname{rank}(d \varkappa)=1\}$. Это множество состоит из восьми лучей, выходящих из одной точки $0=(0,0,0,0)$. Разобьем эти лучи на два класса: $h$-класс, представители которого определяются условием $d f=0$, и $f$-класс, задаваемый условием $d h=0$. На модели $2 f$-крест является прямым произведением точки $0_{1}$ на особый слой $S_{2}^{2}$, соответственно $h$-крест является прямым произведением особого слоя $S_{1}^{2}$ на точку $0_{2}$. Ориентируем лучи векторньм полем $\operatorname{sgrad}_{h}+\operatorname{sgrad}_{f}$. Выделим пару $f$-лучей, удовлетворяющих условию $p_{1}^{k} \geqslant 0, q_{1}^{k} \geqslant 0$. Выделим пару $h$-лучей, удовлетворяюших условию $p_{2}^{k} \geqslant 0, q_{2}^{k} \geqslant 0$. Заметим, что в каждую связную компоненту $G_{f}$ входит или выходит ровно один $f$-луч. Аналогично для $G_{h}$.

Очевидно, что для любого $k L^{k}$ есть стандартная окрестность, т.е. в вершину входит два $f$ - и два $h$-луча и выходит два $f$ - и два $h$-луча.

На каждом ребре графа $L^{*}$ выберем по точке и разрежем по ним. Остов $L^{*}$ распадается на $n$ кусков, каждый из которых гомеоморфен с сохранением ориентации, выделения пар ребер, $f$ - и $h$-классов изображенной на рис. 4 фигуре. Следовательно, $L^{*}$ можно получить из $L^{k}, k=1, \ldots, n$, склейкой и сопоставлением каждому ребру метки $\varepsilon$. Такую операцию назовем базовой склейкой.

Разобьем связные компоненты $G_{h}$ на пары следующим образом. Для каждого $h$-ребра рассмотрим лучи $l_{1}$ и $l_{2}$, из которых это ребро склеено при базовой склейке. Для определенности предположим, что луч $l_{1}$ выходит из некоторой связной компоненты $G_{h}$, а луч $l_{2}$ входит. Выделим эти связные компоненты в одну пару. Легко видеть, что таким образом мы разобьем все связные компоненты $G_{h}$ на па-

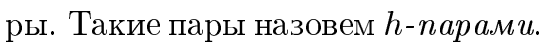

Совершенно аналогично разобьем связные компоненты $G_{f}$ на $f$-napb.

Легко видеть, что в каждую $h$-пару входит связная компонента множества $G_{h+}$ и одна связная компонента $G_{h-}$. Аналогично для $f$-пар.

Паре, определенной по ребру $l$, сопоставим метку $\varepsilon$ этого ребра.

Склеивающие гомеоморфизмы. Для каждой такой пары связных компонент зададим гомеоморфизм между элементами этой пары.

Рассмотрим $h$-пару связных компонент. Удобнее задавать гомеоморфизм, используя модель 2. Каждая связная компонента, входящая в $h$-пару, есть прямое произведение жирно выделенного отрезка границы диска $S_{1}^{2}$ на диск $S_{2}^{2}$. Гомеоморфизм зададим на каждой компоненте прямого произведения. Гомеоморфизм на граничном отрезке выберем таким, чтобы он сохранял функцию $h$. Заметим, что он однозначно определяется этим условием. В качестве гомеоморфизма из $S_{2}^{2}$ в $S_{2}^{2}$ возьмем тождественный, если метка $\varepsilon$ этой пары равна +1 , и центральную симметрию относительно точки $0_{2}$, если метка $\varepsilon=-1$. 
Аналогично зададим гомеоморфизмы между компонентами $f$-пар.

Полученное в результате склейки многообразие обозначим $U_{n}$.

Задание гладкой структуры на $U_{n}$. Точки, отождествленные с помощью построенных выше гомеоморфизмов будем называть точками склейки. Для каждой точки склейки зададим некоторую ее окрестность и координаты в ней такие, что:

1) они являются симплектическими для формы $\omega$;

2) отображение перехода в пересечении двух любых таких окрестностей от одних координат к другим является гладким отображением;

3) отображение перехода в пересечении любого блока с любой такой окрестностью от координат на блокек координатам на окрестности является гладким отображением.

Задав такие координаты, мы докажем теорему, так как, очевидно, $\mathrm{CL}\left(U_{n}, h, f, \omega\right)$ $=\mathbf{L}$.

Пусть $Z \in U_{n}, u$ - гладкое векторное поле на $U_{n}$. Обозначим через $t_{u}(Z)$ минимальное по модулю время, необходимое, чтобы добраться от точки $Z$ до точки склейки, двигаясь вдоль векторного поля $u$. Легко видеть, что $\omega\left(\operatorname{sgrad}_{t_{u}}, u\right)= \pm 1$.

Пусть $X$ - точка склейки. Предположим, что $X$ есть прямое произведение внутренней точки диска $S_{1}^{2}$ на точку отрезка $\alpha$. Рассмотрим координаты $x_{1}, x_{2}, y_{1}, y_{2}$ в малой окрестности точки $X$ такие, что $x_{1}=p_{1}, x_{2}=f, y_{1}=q_{1}, y_{2}=t_{\operatorname{sgrad}_{f}}$. Они действительно являются координатами, так как дифференциал функции $y_{2}$ в точке $X$ не равен нулю из-за того, что векторное поле $\operatorname{sgrad}_{f}$ не обрашается в нуль в точке $X$, и, очевидно, линейно независим с дифференциалами функций $x_{1}, x_{2}, y_{1}$.

Для точек склейки, являющихся прямым произведением точки выделенного отрезка границы $\partial S_{1}^{2}$ на внутреннюю точку диска $S_{2}^{2}$, введем координаты аналогичньм образом.

Для точек склейки, являюшихся прямым произведением точки выделенного отрезка границы $\partial S_{1}^{2}$ на точку выделенного отрезка границы $\partial S_{2}^{2}$, введем координаты следуюшим образом.

Пусть $X$ - такая точка. Рассмотрим координаты $x_{1}, x_{2}, y_{1}, y_{2}$ в малой окрестности точки $X$ такие, что $x_{1}=h, x_{2}=f, y_{1}=t_{\operatorname{sgrad}_{h}}, y_{2}=t_{\text {sgrad }_{f}}$. Покажем, что они действительно являются координатами. Заметим, что до сих пор мы не использовали условие допустимости CL-типа $\mathbf{L}$.

Углом будем называть полудиск с выделенной точкой, называемой верииной, на гранище. Удобно представлять себе угол как множество $\{x \geqslant 0, y \geqslant 0\}$ с вершиной $(0,0)$. Сторонами угла называются $\{x>0\}$ и $\{y>0\}$.

Окрестность точки $X$ в каждом блоке устроена как прямое произведение угла $W$ на интервал $I_{h}$ и на интервал $I_{f}$. Функции $h$ и $f$ являются параметрами на соответствующих интервалах и расслаивают их на точки. Интегральные траектории $\operatorname{sgrad}_{h}$ входят или выходят из одной стороны угла и идут вдоль второй, интегральные траектории $\operatorname{sgrad}_{f}$ входят или выходят из второй стороны угла и идут вдоль первой. Из условия допустимости и из построения следует, что в каждой такой точке $X$ "встречаются" ровно четыре угла, причем склейка и векторные поля $\operatorname{sgrad}_{h}$ и $\operatorname{sgrad}_{f}$ устроены следующим образом. Сторона, в которую входит интегральная траектория $\operatorname{sgrad}_{f}$, и вдоль которой интегральная траектория $\operatorname{sgrad}_{h}$ идет по направлению от вершины (к вершине), склеивается со стороной, из которой 
выходит интегральная траектория $\operatorname{sgrad}_{f}$ и вдоль которой интегральная траектория $\operatorname{sgrad}_{h}$ идет по направлению от вершины (к вершине). Аналогично, сторона, в которую входит интегральная траектория $\operatorname{sgrad}_{h}$ и вдоль которой интегральная траектория $\operatorname{sgrad}_{f}$ идет по направлению от вершины (к вершине), склеивается со стороной, из которой выходит интегральная траектория $\operatorname{sgrad}_{h}$ и вдоль которой интегральная траектория $\operatorname{sgrad}_{f}$ идет по направлению от вершины (к вершине). Следовательно, отображения $x_{1}, x_{2}, y_{1}, y_{2}$ определены корректно. Очевидно, что дифференциалы $x_{1}, x_{2}, y_{1}, y_{2}$ линейно независимы.

Построенные координаты удовлетворяют перечисленным выше требованиям. Теорема реализации доказана.

Проблема перечисления. Теорема реализации дает удобный алгоритм для перечисления всех седловых окрестностей с точностью до топологической эквивалентности. Для перечисления всех седловых окрестностей с точностью до топологической эквивалентности достаточно перечислить допустимые CL-типы. Для веса 1, 2, 3 эта работа была проделана, соответственно, Л. М. Лерманом и Я. Л. Уманским [4], А.В. Болсиновьм [5] и Н. Максимовой. Их результаты можно свести в следующую теорему.

Теорема 2 (о количестве седловых окрестностей веса $1,2,3$ ). C точностью до топологической әквивалентности, существует ровно

4 седловых окрестностей веса 1 ,

39 седловых окрестностей веса 2 ,

256 седловых окрестностей веса 3.

\section{§5. Проблема топологического описания границы седловой окрестности}

Пусть бифуркационная диаграмма седловой окрестности $\mathfrak{U}_{S}$ изображена на рис. 1 в). Рассмотрим окружность $\gamma$ небольшого радиуса $\delta$ с центром в точке $A$. Тогда $\varkappa^{-1}(\gamma)$ может быть описана в терминах инвариантов Фоменко-Цишанга. Полученную меченую молекулу назьвают круговой меченой молекулой точки $A$. Ясно, что топологическое строение границы $\partial \mathfrak{U}_{S}$ седловой окрестности $\mathfrak{U}_{S}$ совпадает с топологическим строением $\varkappa^{-1}(\gamma)$.

Эффективный алгоритм, строящий по CL-типу круговую молекулу, построен А.В. Болсиновым в работе [5]. Мы не будем здесь приводить этот алгоритм, а ограничимся его идеей.

Для удобства считаем, что седловая окрестность склеена из блоков как в предыдушем параграфе. На модели 2 рассмотрим выделенные пунктиром отрезки $\tau_{j}^{i}$ и разрежем каждый блок по подмногообразиям вида: произведение выделенного пунктиром отрезка на $S_{i}^{2}$. Многообразие $U^{4}$ распадается на куски $W_{k}$. Легко видеть, что каждый такой кусок $W_{k}$ является прямым произведением квадрата $Y$ на диск $D^{2}$, диск $D^{2}$ тривиально расслоен на точки функциями $h$ и $f$, единственный слой на квадрате $Y$ совпадает с ним самим. Сторонами квадрата $Y$ являются отрезки вида прямого произведения точки на выделенных отрезках на кусок слоя функции на $S^{2}$ (рис. 3). Отметим, что

1) траектории $\operatorname{sgrad}_{h}$ выходят из ребра квадрата и входят в противоположное ребро, траектории $\operatorname{sgrad}_{f}$ - аналогично; 
2) $Y$-квадраты при значении функций $(f, h)=(0,0)$ являются гранями комплекса $K$.

Каждый слой Лиувилля склеен из таких $Y$-квадратов. С изменением функций $f$ и $h$ эти квадраты "плывут" по многообразию $U^{4}$, переклеиваясь на слоях вида $f=0$ или $h=0$. Алгоритм А.В. Болсинова состоит в формальном описании правил переклеек $Y$-квадратов на особых слоях.

В работе [8] В.С. Матвеев построил с помощью этого алгоритма круговые молекулы точек веса 2. Все они оказались разные. Из работы [3] следует, что круговые молекулы различных седловых окрестностей различны. Следовательно, верна следующая теорема.

ТЕОРема 3. Топологическое строение границы есть полный топологический инвараинт седловой окрестности веса 1 и 2, т.е. две седловые окрестности веса 1 или 2 топологически эквивалентны, если и только если их круговые меченые молекуль одинаковы.

ЗАмЕчАнИЕ. Число $Y$-квадратов и, следовательно, вес окрестности определяются меченой молекулой.

ТЕОрема 4. Топологическое строение гранииы есть полный топологический инвариант седловой окрестности веса 3 , т.е. две седловые окрестности веса 3 топологически эквивалентны, если и только если их круговые меченые молекуль одинаковы.

Доказательство этой теоремы основывается на списке допустимых CL-типов веса 3, включает длинньй перебор и не будет здесь приведено.

\section{§6. Полный топологический инвариант фокусных окрестностей}

Задача этого параграфа состоит в построении удобного полного топологического инварианта для фокусных окрестностей. Напоминаем, что под фокусной окрестностью мы понимаем насьшенную окрестность $\mathfrak{U}_{F}$ точки типа фокус-фокус, не содержащую вырожденных точек, бифуркационная диаграмма которой состоит из одной изолированной точки $A$, прообраз которой связен.

Легко видеть, что все особые точки слоя $K=\varkappa^{-1}(A)$ являются точками ранга 0 и имеют тип фокус-фокус. Действительно, бифуркационная диаграмма и невырожденной точки ранга 1 , и невырожденной точки ранга 0 типа, отличного от типа фокус-фокус, содержит по крайней мере интервал.

Это означает, в частности, что в слое $K$ не содержится одномерных орбит, и слой $K$ представляет собой склейку некоторого числа колец $O=S^{1} \times I$, плоскостей $P^{2}$ и точек. Компонентами склейки являются орбиты.

Пусть $N\left(\mathfrak{U}_{F}\right)$ - количество точек типа фокус-фокус на слое $K$.

ТЕОРема 5. Число $N\left(\mathfrak{U}_{F}\right)$ - полный реализуемый топологический инвариант фокусной окрестности.

Докажем сначала пару лемм. Как и в $\S 3$, считаем окрестность $\mathfrak{U}_{F}$ достаточно тонкой. 
ЛЕмма 1 (о разбиении). Пусть слой $K$ является склейкой $N\left(\mathfrak{U}_{F}\right)$ точек типа фокус-фокус, $m_{K}$ двумерных орбит, гомеоморфных кольиу, и $m_{3}$ двумерных орбит, гомеоморфных плоскости. Тогда окрестность $\mathfrak{U}_{F}$ можно разрезать на $N\left(\mathfrak{U}_{F}\right)+m_{3}$ кусков, $N\left(\mathfrak{U}_{F}\right)$ из которых топологически әквивалентны $F=\left\{D^{4}, h, f\right\}$, где $D^{4}=\left\{x_{1}^{2}+y_{1}^{2}+x_{2}^{2}+y_{2}^{2}<1,\left|x_{1} y_{1}+x_{2} y_{2}\right|<1 / 4\right.$, $\left.\left|x_{1} y_{2}-x_{2} y_{1}\right|<1 / 4\right\}$ - четырехмерный диск, $h=x_{1} y_{1}+x_{2} y_{2}$ u $f=x_{1} y_{2}-x_{2} y_{1}$ - функции, задающие слоение; $m_{3}$ из которых топологически әквивалентны $D^{2} \times P^{2}$, слоем на $P^{2}$ считаем любую точку, единственный слой на диске $D^{2}$ совпадает с ним самим.

Доказательство этой леммы полностью аналогично доказательству леммы 3 из $\S 3$. Для каждой особой точки типа фокус-фокус $p_{i}$ рассмотрим ее окрестность $F_{i}$, топологически эквивалентную $F$. Окрестность $\mathfrak{U}_{F}$ считаем такой тонкой, что пересечение $\mathfrak{U}_{F}$ с любым $F_{i}$ содержится в этом $F_{i}$, под окрестностью $F_{i}$ будем в дальнейшем понимать это пересечение.

Рассмотрим поподробнее $F$. Особый слой $F$ гомеоморфен паре пересекающихся по одной точке двумерных плоскостей (удобней представлять как пару конусов с обшей вершиной). Каждый неособый слой гомеоморфен кольцу $O=S^{1} \times I$. Объединение границ всех слоев является двумя тривиально расслоенньми на окружности полноториями. Одна граничная окружность каждого слоя лежит на одном полнотории (скажем, $\left.P_{1}^{3}\right)$, другая на другом $\left(P_{2}^{3}\right)$.

Легко видеть, что пересечение $F_{i}$ с любой двумерной орбитой является регулярной окрестностью связной компоненты границы этой орбиты. Для каждой двумерной орбиты $\theta^{2}$, лежащей на слое $K$, рассмотрим разность $\widehat{\theta}=\theta^{2} \backslash \bigcup_{i} F_{i}$. Очевидно, что $\widehat{\theta}$ гомеоморфно $\theta^{2}$. По лемме $5 \S 3$ сушествует окрестность $W_{j}^{4}$, топологически эквивалентная $\widehat{\theta} \times P^{2}$, слоем на $P^{2}$ считаем любую точку. Можно выбрать эту окрестность так, что соответствующие граничные полнотория соседних с $W_{j}$ $F$-окрестностей совпадают с соответствуюшими граничными полноториями $W_{j}$. Для доказательства леммы осталось добавить, что присоединение $W$-окрестностей типа $O \times P^{2}$ к граничащим с ними $F$-окрестностями не меняет топологическую структуру последних.

Такое разбиение будем называть базовым, для каждой фокусной окрестности фиксируем ее некоторое базовое разбиение. Следующая лемма утверждает, что на слое $K$ нет $\mathbb{R}^{2}$-орбит.

ЛЕмма 2. $m_{3}=0$.

Рассмотрим $n$ двумерных сфер. Выберем по две точки на каждой сфере. Выбранные точки на $i$-ой сфере будем обозначать $A_{i}$ и $B_{i}$. Склеим $A_{i}$ с $B_{i+1}$ для каждого $i \in\{1,2, \ldots, n+1\}$. Результат склейки обозначим через $C_{H}$. Склеим $A_{n}$ с $B_{1}$. Результат склейки обозначим через $C_{3}$. Топологическое пространство будем называть незамкнутой цепью, если оно гомеоморфно $C_{H}$, и замкнутой цепью, если оно гомеоморфно $C_{3}$.

ДокАЗАТЕЛЬСтво ЛЕммы 2. Слой $K$ склеен из точек типа фокус-фокус, колец и дисков. Около точки типа фокус-фокус слой $K$ устроен как пара конусов с общей вершиной. Используя лемму 1 и связность $\mathfrak{U}_{F}$, получаем, что $K$ либо замкнутая, либо незамкнутая цепь. Легко видеть, что во втором случае все неособые слои 
гомеоморфны сфере, что является противоречием с теоремой Лиувилля. Лемма доказана.

ДоказАТЕЛЬСтво теоремы 5. Рассмотрим базовое разбиение. Пусть $F_{i}$ склеивается с $F_{i+1}, i \in \mathbb{Z}_{N\left(\mathfrak{U}_{F}\right)}$, по полноторию $P_{i}$. Напоминаем, что $\mathbb{Z}_{N\left(\mathfrak{U}_{F}\right)}$ обозначает группу вычетов по модулю $N\left(\mathfrak{U}_{F}\right)$. Назовем ориентацию слоев граничных полноторий окрестности $F$ согласованной, если для любого неособого слоя-кольца ориентация его граничных окружностей согласована.

На каждом полнотории $P_{i}, i \neq 0$, ориентируем слои граничных полноторий следующим образом. На $P_{1}$ ориентируем произвольно, на $P_{2}$ ориентируем так, чтобы ориентация слоев граничных полноторий окрестности $F_{2}$ была согласована (это можно сделать единственным способом), и т. д.

УТВЕРЖДЕНИЕ 1. Ориентация слоев граничных полноторий окрестности $F_{1}$ согласована.

ДоКАЗАТЕЛЬСтво. Предположим противное. Выберем какой-нибудь неособый слой $Y$ окрестности $\mathfrak{U}_{F}$. По теореме Лиувилля он гомеоморфен двумерному тору. С другой стороны, он является склейкой из $N\left(\mathfrak{U}_{F}\right)$ колец по граничным окружностям, причем ровно один склеивающий гомеоморфизм меняет ориентацию. Следовательно, $Y$ гомеоморфен бутылке Клейна. Противоречие.

Итак, пусть $\mathfrak{U}_{F}$ и $\widehat{\mathfrak{U}}_{F}$ - две фокусные окрестности, $N\left(\mathfrak{U}_{F}\right)=N\left(\widehat{\mathfrak{U}}_{F}\right)$. Рассмотрим их базовые разбиения. Обе эти окрестности склеены из одинаковых кусков. Соответствуюшие гомеоморфизмы склеек гомотопны в силу утверждения 1 леммы $4 \S 3$ и утверждения 1 этого параграфа. Легко видеть, что если слой $X$ окрестности $F$ склеился со слоем $Y$, соответствующий слою $X$ слой $\widehat{X}$ окрестности $\widehat{F}$ склеился со слоем $\widehat{Y}$, то $Y$ гомеоморфен $\widehat{Y}$. Следовательно, сушествует сохраняющий слоение Лиувилля гомеоморфизм между $\mathfrak{U}_{F}$ и $\widehat{\mathfrak{U}}_{F}$.

Мы получили, что если $N\left(\mathfrak{U}_{F}\right)=N\left(\widehat{\mathfrak{U}}_{F}\right)$, то $\mathfrak{U}_{F}$ и $\widehat{\mathfrak{U}}_{F}$ топологически эквивалентны. Для доказательства теоремы осталось доказать лемму реализации.

ЛЕмма 3 (лемма реализации). Для любого натурального числа $n$ существует фокусная окрестность $\mathfrak{U}_{F}$ веса $N\left(\mathfrak{U}_{F}\right)=n$.

ДокАЗАТЕльство. Для $n=1$ эта лемма верна, так как фокусная окрестность $\mathfrak{U}_{1}$ веса $N\left(\mathfrak{U}_{1}\right)=1$ встретилась в случае Лагранжа движения твердого тела (см., например, [7]). Используя $\mathfrak{U}_{1}$, построим фокусную окрестность $\mathfrak{U}_{n}$ веса $n$.

Рассмотрим базовое разбиение фокусной окрестности $\mathfrak{U}_{1}$. Возьмем $n$ копий получившейся в результате этого разбиения окрестности $F$. Обозначим их $F_{i}, i=$ $1, \ldots, n$. Граничные полнотория $i$-й копии обозначим через $P_{1, i}$ и $P_{2, i}$. Склеим $P_{1, i}$ и $P_{2, i+1}, i \in \mathbb{Z}_{n}$, по гомеоморфизму, получаемому как композиция гомеоморфизма, склеивающего базовое разбиение окрестности $\mathfrak{U}_{1}$ в $\mathfrak{U}_{1}$, и “тождественных" гомеоморфизмов между окрестностью $F$ и ее копиями $F_{i}$ и $F_{i+1}$. На полученном в результате склейки многообразии $\mathfrak{U}_{n}$ зададим структуру дифференцируемого многообразия так же, как она была задана при склейке компоненты базового разбиения окрестности $\mathfrak{U}_{1}$ многообразия $F$ в окрестность $\mathfrak{U}_{1}$. Функции $h, f$ и форма $\omega$ склеиваются гладко, так как они склеивались гладко при склейке $F$ в $\mathfrak{U}_{1}$. Следовательно, $\left(\mathfrak{U}_{n}, \omega, h, f\right)$ - интегрируемая гамильтонова система. Легко видеть, 
что $\mathfrak{U}_{n}$ - фокусная окрестность, на особом слое которой лежит ровно $n$ точек типа фокус-фокус, что и требовалось доказать.

СлЕДСТВИЕ 1. Существует алгоритм, который по двум фокусным окрестностям определяет, әквивалентны ли они.

Этот алгоритм состоит в подсчете числа точек типа фокус-фокус.

СлЕДСТВИЕ 2. Существует алгоритм, перечисляющий фокусные окрестности с точностью до топологической эквивалентности.

Следствия 1 и 2 решают проблемы распознавания и перечисления.

Проблема описания топологического строения границы. Рассмотрим окружность $\gamma$ с центром в точке $A$. Очевидно, что ее прообраз $Q^{3}=\varkappa^{-1}(\gamma)$ топологически эквивалентен границе фокусной окрестности $\mathfrak{U}_{F}$. При выбрасьвании любой точки этой окружности прообраз полученного интервала топологически эквивалентен по теореме Лиувилля прямому произведению двумерного тора $T^{2}$ на интервал, слоем на интервале считаем любую точку. Следовательно, $Q^{3}$ топологически эквивалентно такому прямому произведению со склеенными граничньми торами. Выберем базис на торе $T^{2}$. Он индуцирует базис на граничных то$\operatorname{pax} T_{1}$ и $T_{2}$ прямого произведения тора на интервал. Гомеоморфизм из $T_{1}$ в $T_{2}$, с точностью до гомотопии, задается в этом базисе целочисленной обратимой $2 \times 2$ матрицей $E$. При выборе другого базиса матрица $E^{\prime}$, задающая гомеоморфизм в новом базисе, связана с $E$ следующим соотношением: $E=J E^{\prime} J^{-1}$, где $J$ - матрица перехода из нового базиса в старый. Следовательно, топологическое строение границы задается классом целочисленных обратимых $2 \times 2$ матриц по отношению эквивалентности сопряжению целочисленньми обратимыми $2 \times 2$ матрицами.

ЛЕмма 4. Класс матрии, задающих топологическое строение границъ $Q_{n}^{3}$ фокусной окрестности $\mathfrak{U}_{n}$ веса $N\left(\mathfrak{U}_{n}\right)=n$, имеет представителем матрицу $E_{n}=\left|\begin{array}{ll}1 & n \\ 0 & 1\end{array}\right|$.

ДоКАЗАТЕЛЬСТвО. Проверка леммы для $n=1$ состоит в достаточно сложном технически, но идейно простом подсчете. Достаточно реализовать $\mathfrak{U}_{1}$ как склейку граничных полноторий в $F^{4}=\left\{D^{4}, h, f\right\}$, где $D^{4}=\left\{x_{1}^{2}+y_{1}^{2}+x_{2}^{2}+y_{2}^{2}<1\right.$, $\left.\left|x_{1} y_{1}+x_{2} y_{2}\right|<1 / 4,\left|x_{1} y_{2}-x_{2} y_{1}\right|<1 / 4\right\}$ - четырехмерный диск, $h=x_{1} y_{1}+$ $x_{2} y_{2}$ и $f=x_{1} y_{2}-x_{2} y_{1}-$ функции, задаюшие слоение, с помощью гомеоморфизма $\varphi: T_{1} \rightarrow T_{2}$, определяемого по правилу:

$$
\varphi\left(p_{1}, q_{1}, p_{2}, q_{2}\right)=\left(\alpha p_{1}, q_{1} / \alpha, \alpha p_{2}, q_{2} / \alpha\right), \quad \alpha=\frac{\sqrt{\rho_{q_{1}^{2}+q_{2}^{2}}}}{\sqrt{\rho_{p_{1}^{2}+p_{2}^{2}}}}
$$

Базис на торах можно выбрать, например, так. В качестве параллелей и меридианов возьмем кривые

$$
\mu=\left(a e^{\varphi}, b e^{\varphi}, c e^{-\varphi}, d e^{-\varphi}\right)
$$

и

$\lambda=(a \sin (\varphi)+b \cos (\varphi), c \sin (\varphi)+d \cos (\varphi), b \sin (\varphi)-a \cos (\varphi), d \sin (\varphi)-c \cos (\varphi))$, 
где $a^{2}+b^{2}+c^{2}+d^{2}=1$.

Кривая $\mu$ гомеоморфна окружности, так как отображение $\varphi$ отождествляет ее концевые точки. В этом базисе матрица склейки $E_{1}=\left|\begin{array}{ll}1 & 1 \\ 0 & 1\end{array}\right|$.

Для доказательства леммы в случае произвольного веса $n$ рассмотрим базовое разбиение окрестности $\mathfrak{U}_{n}$. Склеим окрестность $U_{n}$ из компонент этого разбиения $F_{i}^{4}$ следуюшим образом. Рассмотрим на $i$-ой компоненте функцию $\widehat{f}=f+\varepsilon_{i}$, все $\varepsilon_{i}$ малы и различны. Склеим $F_{i}^{4}$ в $U_{n}$ по тем же полноториям, что и в $\mathfrak{U}_{n}$, но с сохранением функций $h$ и $f$. Удалим все незамкнутые слои. Очевидно, что граница $U_{n}$ топологически эквивалентна границе $\mathfrak{U}_{n}$. С другой стороны, видно, что склеивающий гомеоморфизм граничных торов является $n$-й степенью склеивающего гомеоморфизма для граничных торов границы $Q_{1}^{3}$. Для доказательства леммы осталось проверить, что $E_{1}^{n}=E_{n}$, а это очевидно.

СледСтвиЕ. Для фокусньх окрестностей проблема Фоменко верна.

\section{§7. Некоторые замечания}

Круговую молекулу $W^{*}$ назовем реализуемой, если сушествует такая не содержашая вырожденных точек интегрируемая гамильтонова система и такая простая замкнутая кривая $\gamma$ на ее бифуркационной диаграмме, что молекула $W^{*}\left(\varkappa^{-1}(\gamma)\right)=W^{*}$.

Можно показать, что реализуемые круговые молекулы образуют разрешимое множество, т.е. сушествует алгоритм, отвечающий на вопрос, реализуема ли молекула или нет. Всевозможные круговые молекулы седловых окрестностей веса 1 и 2 построены, соответственно, в [5] и [8]. Заметим, что разрешающий алгоритм для круговых молекул достаточно сложный, и мы полагаем, что CL-тип дает более удобный способ хранения информации о седловых окрестностях.

Проблема Фоменко. Из $\S 4,5$ следует, что круговое меченое слово-молекула есть полный топологический инвариант фокусных окрестностей и седловых окрестностей веса 1,2 и 3. Из работ [4]-[6], [11], [12] следует, что аналогичный факт верен и для насьщенных окрестностей точек типа центр-центр и центр-седло. Назовем хорошими интегрируемыми гамильтоновыми системами интегрируемые гамильтоновы системы, удовлетворяюшие следующим условиям:

a) все особые точки невырождены,

б) у любой точки типа седло-седло есть седловая окрестность,

в) у любой точки типа фокус-фокус есть фокусная окрестность,

г) любая невырожденная изоэнергетическая поверхность устойчива.

Теорема (о проблеме Фоменко). Для хороших систем проблема Фоменко верна.

Эта теорема следует из теорем 1 и $2 \S 4$, следствия основной теоремы $\S 5$, из работы [5] и из того, что круговые молекулы точек различного типа невозможно перепутать.

CL-типы, встречающиеся в реальных системах. Точки типа седло-седло возникают при описании движения в так называемых

а) случае Стеклова и случае Клебша, 
б) случае Ковалевской,

в) случае Горячего-Чаплыгина.

Соответствующие CL-типы изображены на рис. 2. Фокусные окрестности встречаются в случае Лагранжа, их вес равен 1 (см. [7]).

Если невозможно представить линии бифуркационной диаграммы в виде графиков гладких функций.

Седловой в широком смысле окрестностью будем назьвать интегрируемую гамильтонову систему $\left(U^{4}, \omega, h, f\right)$

1) не содержащую вырожденных точек,

2) бифуркационная диаграмма которой состоит из пары пересекающихся (по точке $A$ ) интервалов,

3) прообраз точки $A$ связен, $U^{4}$ является регулярной окрестностью прообраза точки $A$.

Две седловые в широком смысле окрестности будем называть топологически әквивалентными, если между ними сушествует гомеоморфизм, сохраняющий слоение Лиувилля.

Рассмотрим диффеоморфизмы плоскости $\mathbb{R}^{2}$, переводящие бифуркационную диаграмму в пару пересекающихся интервалов $\{f h=0,|f|<1,|h|<1\}$. Седловая в широком смысле окрестность при таком отображении превращается в $(+)$ седловую окрестность. Два CL-типа будем считать әквивалентнымии, если от некоторого представителя первого CL-типа можно перейти к представителю второго с помощью операций смены ориентации на всех ребрах белого графа и смены ориентации на всех ребрах черного графа. Фактор множества всех CL-типов по этому отношению эквивалентности будем обозначать $\mathfrak{\mathfrak { L }}$. Отображение из множества седловых в обобшенном смысле окрестностей в множестве $\widehat{\mathfrak{L}}$, сопоставляющее седловой в широком смысле окрестности элемент множества $\widehat{\mathfrak{L}}$ с представителем CL-типом от соответствующей $(+)$ седловой окрестности, назовем обобщенныц CL-отображением. Обобщенное CL-отображение есть полный топологический инвариант седловых в широком смысле окрестностей.

Более сложные пересечения на бифуркационной диаграмме. Если на слое лежит всего одна точка ранга 0 , причем ее тип - седло-седло, то тогда бифуркационная диаграмма тонкой насышенной окрестности этой точки является крестом, и у этой точки есть седловая в широком смысле окрестность. Если на слое есть несколько точек типа седло-седло, то кресты, соответствующие каждой точке, как-то налагаются. Наложения могут быть достаточно сложными. В данной работе мы не затрагивали случай нетривиального наложения, так как в интегрируемых гамильтоновых системах, возникающих при исследовании задач физики и механики, таких случаев нет. Всю теорию CL-типа можно обобщить на случаи более сложных наложений. Действительно, леммы $1,2,3,4,5 \S 3$ переносятся без изменения. Более сложный вид приобретает меченый остов (раскраска не на два, а на три и более число цветов), чуть усложняются отношения эквивалентности и условия допустимости. Даже теорема реализации идейно повторяет теорему реализации этой работы.

Гомео и диффео. Из теории CL-типа очевидно следует, что две топологически эквивалентные системы гладко топологически эквивалентны. (То есть сушест- 
вование гомеоморфизма, сохраняюшего слои Лиувилля, влечет за собой сушествование диффеоморфизма, сохраняющего слои Лиувилля.) Отметим, что аналогичный факт верен и для топологического строения устойчивых невырожденных изоэнергетических поверхностей.

\section{Список литературы}

1. Фоменко A. T. Топология поверхностей постоянной энергии интегрируемых гамильтоновых систем и препятствия к интегрируемости // Изв. АН СССР. Сер. матем. 1986. T. 50. №6. C. 1276-1307.

2. Фоменко A. T., Цишанг X. Критерий топологической эквивалентности интегрируемых гамильтоновых систем с двумя степенями свободы // Изв. АН СССР. Сер. матем. 1990. Т. 54. № 3. С. 546-575.

3. Лерман Л. М., Уманский Я. Л. Структура пуассонова действия на четырехмерном симплектическом многообразии. Горький: Горьковский университет, 1981. Деп. в ВИНИТИ 10.07.81, № 3427 .

4. Лерман Л.М., Уманский Я.Л. О классификации четырехмерных интегрируемых систем в расширенных окрестностях простых особых точек // Методы качественной теории и теории бифуркаций. Межвузовский тематический сборник научных трудов / ред. М.И. Карпович. Горький: Горьковский университет, 1988.

5. Bolsinov A. V. Methods of Calculations of the Fomenko-Zieschang Invariant // Adv. Soviet Math. 1991. V. 6. P. 147-183.

6. Лерман Л. М., Уманский $Я$. Л. Классификация четырехмерных интегрируемых гамильтоновых систем и пуассоновских действий $\mathbb{R}^{2}$ в расширенных простых окрестностях особых точек. I // Матем. сб. 1992. Т. 183. №12. С. 141-176.

7. Oshemkov A. A. Fomenko invariant for the Main Integrable Cases of teh Rigid Body Motion Equations // Adv. in Soviet Math. 1991. V. 6. P. 67-146.

8. Матвеев В. С. Вычисление значений инварианта Фоменко для точки типа седло-седло интегрируемой гамильтоновой системы // Труды семинара по векторному и тензорному анализу с их приложениями к геометрии, механике и физике. № 24, 1993. С. 75-104.

9. Болсинов A.B., Матвеев С.В., Фоменко А.Т. Топологическая классификация интегрируемых гамильтоновых систем с двумя степенями свободы. Список систем малой сложности // УМН. 1990. Т. 45. № 2. С. 49-77.

10. Eliasson $L$. $H$. Normal form Hamiltonian systems with Poisson commuting integral-elliptic case // Comm. Math. Helv. 1990. V. 65. P. 4-35.

11. Лерман Л.М., Уманский Я.Л. Классификация четырехмерных интегрируемых гамильтоновых систем и пуассоновских действий $\mathbb{R}^{2}$ в расширенных простых окрестностях особых точек. II // Матем. сб. 1992. Т. 183. № 12. С. 141-176.

12. Лерман Л.М., Уманский Я.Л. Классификация четырехмерных интегрируемых гамильтоновых систем и пуассоновских действий $\mathbb{R}^{2}$ в расширенных окрестностях простых особых точек. III. Реализация // Матем. сб. 1995. Т. 186. № 10. С. 89-102.

Московский государственньй

Поступила в редакцию

университет им. М.В. Ломоносова

25.10 .1995 